\title{
Essai sur la faune cavernicole de Bulgarie. III. Résultats des recherches biospéologiques de 1966 à 1970
}

\author{
P. BERON*
}

\section{INTRODUCTION}

Dix ans se sont écoulés depuis la parution de notre premier travail de cette série**. Le développement rapide des recherches sur le domaine souterrain nous a incités à rédiger cinq ans plus tard une liste supplémentaire (Beron et Guéorguiev, 1967), qui, elle aussi, n'est plus à jour.

L'idée de mettre au point tous les 5 ans les connaissances sur la faune cavernicole bulgare ayant trouvé un bon accueil par les spécialistes, nous avons préparé ce nouveau supplément. Il contient des données (souvent inédites) sur 283 grottes et gouffres bulgares, dont 148 ne figurent ni dans notre liste de $1962 \mathrm{ni}$ dans celle de 1967. Cela fait que l'on connaît à présent 431 cavités karstiques en Bulgarie dont la faune est plus ou moins connue. Plus de 400 d'entre elles ont été explorées par l'auteur personnellement. On trouvera ici une énumération de 293 espèces animales, dont 154 ne sont pas mentionnées dans les deux listes précédentes.

Cet accroissement sensible de nos connaissances sur la faune cavernicole bulgare est due à l'activité de quelques récolteurs passionnés et de plusieurs spécialistes bulgares et étrangers qui ont étudié d'une manière approfondie le matériel confié. S'il faut citer quelques noms des spéléologues bulgares, qui ont récol té ce matériel dans des conditions souvent difficiles, nous donnerons en priorité ceux de MM. V.Beškov, Hr.Delčev, St.Andreev, A.Popov, P.Tranteev et autres. Dans la période étudiée relativement peu de chercheurs étrangers ont effectué des récoltes en Bulgarie. Il y a lieu d'en citer cependant: MM.P.Browne et W.G.R.Maxwell (Angle terre) et W.Staręga (Pologne).

$\mathrm{Au}$ cours de nos prospections nous avons profité, une fois de plus, des conseils du Dr. I.Buresch, Membre de l'Académie des Sciences de Bulgarie, Nestor de la Biospéléologie bulgare.

Nous sommes redevables à l'excellent spéléologue bulgare, M.P.Tranteev, qui nous a fait bénéficier de ses vastes connaissances sur les grottes en Bulgarie. Nous avons emprunté une bonne partie des données sur les dimensions, la situation etc. des grottes énumérées du Fichier général des grottes bulgares dont M.Tranteev est le responsable. Nous sommes redevables aussi à M.V.Guéorguiev qui nous a fait part de ses avis concernant certains problèmes biospéléologiques.

Dans la période prospectée ont eu lieu des études approfondies sur plusieurs groupes d'animaux cavernicoles bulgares. Aux auteurs comme MM. H.Coiffait

\footnotetext{
* Institut de Zoologie, 1 Boulevard Ruski, SOFIA, Bulgarie

** Guéorguiev V. et Beron P., 1962, Essai sur la faune cavernicole de Bulgarie, Annales de Spéléol., XVII, 2 et 3, p. 285-441.
} 
(Toulouse), A.Riedel (Warszawa), K.Strasser (Trieste), I.Tabacaru (Bucarest), A.Vandel (Toulouse) et autres nous devons des contributions précieuses apportées à l'étude de cette fauna, Quelques articles intéressants sont dûs aussi à nos collègues bulgares, MM. St.Andreev, V.Bešovski, V.Guéorguiev, Hr.Delčev et autres. Les résultats de l'expédition anglaise en Bulgarie (1967) ont été mis au point par Mme M.Hazelton.

Le présent article contient aussi plusieurs données inédites. Nous sommes bien reconnaissants aux collègues, qui nous ont fait bénéficier de leurs déterminations, et notamment: Mme M.Gruia (Collembola) et MM. St.Andreev (Amphipoda et Isopoda), N.Atanasov (Formicidae) H.Coiffait (Coleoptera), Hr.Delčev (= Deltshev) (Araneida), I.Dlabola (Homoptera), V.Guéorguiev (Coleoptera), A.Riedel (Gastropoda), K.Strasser (Diplopoda), J.-M.Thibaud (Collembola) et A.Wiktor (Gastropoda).

\section{Quelques mots sur le développement de la spéléologie bulgare de 1961 à 1970}

Les prospections, effectuées dans plusieurs régions karstiques de Bulgarie ces 10 dernières années ont fait changer beaucoup nos idées quant à la répartition et aux dimensions des grottes et des gouffres bulgares. A présent toutes les régions karstiques de notre pays sont prospectées dans leurs grandes lignes, grâce surtout à l'activité des spéléoclubs bulgares. Parmi les clubs les plus actifs il y a lieu de citer les Clubs des Sociétés de tourisme "Akademik", "Edelweiss" et "Planinec" de Sofia, les Spéléoclubs de Tchepelare (Čepelare), Vratza (Vraca), Tolbuhin, Trojan etc. Une étude systématique des grottes bulgares est en cours, effectuée par P.Tranteev et par l'auteur de cet article.

Grottes bulgares dont la longueur totale dépasse $1000 \mathrm{~m}$.:

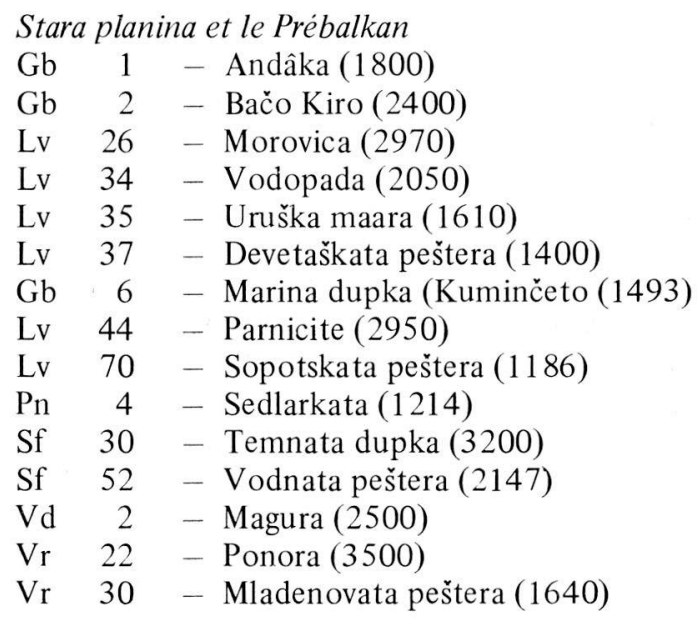




\section{La Bulgarie au Nord du Prébalkan}

Rs 1 - Orlova čuka (plus de 10000)

Šn $6-$ Zandana (1800)

Vitoša

Pk 1 - Duhlata (plus de 8500)

Rhodopes

Sm 1 - Lednicata (1419)

Sm $2-$ Hvojnenskata peštera (1200)

Sm 6 - Imamova dupka (7000)

\section{PARTIE SYSTÉMATIQUE}

\section{Mollusca Gastropoda}

Fam. Z o n i t i d a e

Oxychilus (Morlina) glaber striarius (Westerlund, 1881) (A.Riedel det.)

K1 6 - Meča dupka, 27.II.1969, P.Beron et V. Bešk ov leg. (“Uebergang zu O.glaber nitidissimus (Mousson, 1859), 9 juv. ex.”). Lv 44 - Parnicite, 16.II.1963, V.Beškov et P.Beron leg. Lv 64 - Malkata Červenska jama, 24.XI.1968, P.Beron leg. Lv 66 - Svinštica, 18.V.1968, id. leg. Lv 70 - Sopotskata peštera, 24.X.1965, V.Beškov et W.Staręga leg. Mg 3 - Vreloto, 31.I.1961, P.Beron leg. Mg 16 - Dupkata, 4.II.1967, P.Beron et V.Beškov leg. Mg 21 - Srednjata, 11.X.1969, P.Beron leg. Mg 22 - Gornjata, 11.X.1969, id. leg. Pv 5 - Ledenicata, 6.VII.1962, id. leg. Pv 9 - Erkjuprija, 18.III.1968, P.Beron et V.Beškov leg. Sf 40 - Jamata, 14.IX.1957, T.Uminski leg. Sm 10 -Vaklite dupki,.VII.1962, P.Beron et St.Andreev leg. Šn 8 - Lozevskata peštera, 22.IX.1968, P.Beron leg. Vd 1 - Vârkan, 14.X.1969, id. Vd 18 - Studena, 15.X.1969, id. Vd 21 - Prelaz, 21.X.1969, id. Vd 33 - Koritska glama, 22.X.1969, id. Vd 35 - Carevica, 22.X.1969, id. Vr 3 - Studenata dupka, 4.XII.1924, I.Buresch leg.

Troglophile.

Oxychilus (Morlina) urbanskii Riedel, 1963

Ja 14 - Leleška dupka 3, le 8.VIII.1970, P.Beron et V.Beškov leg. (A.Riedel det., "nicht ganz typisch").

Troglophile (?).

Oxychilus (Oxychilus) hydatinus (Rossmaessler, 1838)

Ja 15 - Jamata, 9.VIII.1970, P. Beron et V.Beškov leg. (A.Riedel det., avec “? ”).

Troglophile (?).

Oxychilus (Riedelius) depressus (Sterki, 1889)

Lv 20 - Rušovata peštera (Riedel, 1969, p.111). Vr 35 - Propastta (id.). Sl 3 - Gornata maaza (id., p.107). Sz 3 - Toplata dupka (id.)

Trogloxène.- Peut être troglophile. Riedel (1969, p.111) est d'avis que "ist es nicht 
ausgeschlossen, dass in den Höhlen des südwestlichen Rumäniens und möglicherweise auch in Bulgarien ausschliesslich die grosse Form von O.depressus (Sterki) vorkommt".

Oxychilus (Schistophallus) moussoni (Kobelt, 1878)

Bs 6 - Izvornata peštera, 9.VIII.1970, P.Beron et V.Beškov leg. (A.Riedel det.).

Troglophile (?).

Vitrea diaphana (Studer, 1820)

Sf 74 - Padež, 1.VI.1969, P.Beron leg. (A.Riedel det.).

Trogloxène.- Troisième trouvaille en Bulgarie.

Daudebardia rufa (Draparnaud, 1805)

Lv 5 - Bankovica (Riedel, 1967, p. 469). Mg 16 - Dupkata, 1e 4.11.1967, P. Beron et V. Beškov leg. (A.Riedel det). Vr 2 - Drašanskata peštera, 8.II.1964, P. Beron leg. (id. det.).

Trogloxène.

Daudebardia brevipes (Draparnaud, 1805)

Vr 38 - Bulina dupka, 2.X.1968, P.Beron leg. (A.Riedel det.).

Trogloxène.

Daudebardia wiktori Riedel, 1967

Pv 9 - Erkjuprija, 18.III.1968, P.Beron et V.Beškov leg. (A.Riedel det.).

Trogloxène.- "5-ter Fund im allgemeinen, erster in einer Höhle" (Riedel, in litt.).

Daudebardia sp.

Vr 58 - Moravata (Popov, 1969, p.37).

Fam. V e r t ig in i d a e gen.sp.

Bs 7 - Kirečnicata, 7.XII.1963, P. Beron leg. (A.Riedel det.).

Fam. L i m a c i d a e

Lytopelte (Lioly topelte) bureschi (H.Wagner, 1934)

Sf 74 - Padež, 1.VI.1969, P. Beron leg. (A.Wiktor det.).

Trogloxène.

Fam. M i 1 a c i d a e

Milax (M.) kusceri H.Wagner, 1931 
Sf 30 - Temnata dupka (Urbanski \& Wiktor, 1967, p.86, sub "Lakatnik... in einer Höhle”). Trogloxène.

N o t e. Dans l'énumération des animaux, récoltés par l'expédition anglaise en 1967 (Hazelton, 1970), on voit aussi quelques Gastropodes, dont la présence dans les deux gouffres bulgares est purement occasionelle. Ce sont:

Lv 5 - Bankovica: Atopia (Attica) quiccardi Roth., Clausilia (Delima) sp. et Monachà haussknechti

Pv 14 - Ivanova voda Chondrula sp. (cf.murotraga), Xerophila aberrans (Mousson), Clausilia sp. et Petraeus ou Ena sp. (Det.Rev.H.E.J. Biggs)

\section{Arthropoda Crustacea Copepoda}

Fam. C a n thoc a m p tid a e

Elaphoidella angelovi Michailova-Neikova, 1969

Sm 20 - Gorna Karanska dupka (Michailova-Neikova, 1969, p.62).

Troglobie.-- Quelques exemplaires ont été récoltés dans la vase des gours à l'intérieur de la grotte. L'espèce semble être proche d'Elaphoidella incerta Chappuis d'une grotte près de Skopje.

$$
\text { Fam. C y c l o p i d a e }
$$

Acanthocyclops viridis (Jurine, 1820)

Lv 5 - Bankovica (Hazelton, 1970, p.33):

Trogloxène régulier.

\section{Amphipoda}

Fam. G a m m a ri d a e

Niphargus bureschi Fage, 1926

Vr 2 - Drašanskata peštera (Popov, 1969, p.40). Vr 44 - Popovata peštera (id., p.38). Vr 57 Starata prodânka (id.). Vr 58 - Moravata (id. p.37). Vd 11 - Golemi pec (Andreev, 1971, p.62). Lv 58 - Skravenika, 13.VII.1963, P.Tranteev leg. (St.Andreev det.). Pn 6 - Gornoto ezero, 19.X.1968, id. leg. (id. det.). Vr 53 - Beljar, 7.X.1968, id. leg. (id. det.). Vr 66 Kondžova kruša, 18.VI.1971, id. leg. (id. det.). Vr 67 - Zvănkova dupka, 2.X.1968, id. leg. (id. det.).

Troglobie. 
Niphargus tauri pecarensis St.\& G.Karaman, 1959

Vd 11 - Golemi peč (Andreev, 1972, p.62).

Troglobie.

Niphargus ablaskiri georgievi St. et G. Karaman, 1959

Lv 30 - Stâlbica, le 9.VII.1968, Hr.Delčev leg. (St.Andreev det.)

Troglobie.

Niphargus sp.

Lv 5 - Bankovica (Hazelton, 1970, p.33). Vr 44 - Popovata peštera (Popov, 1969, p.39).

\section{Isopoda}

Fam. Cirola nid a e

Sphaeromides polateni Angelov, 1968

Lv 78 - Izvora (Angelov, 1968, p.195).

Troglobie.- La quatrième espèce connue du genre Sphaeromides

Sphaeromides bureschi Strouhal, 1963

Vr 53 - Beljar, le 7.X.1968, plusieurs ex., P.Beron leg. (St.Andreev det.).

Troglobie.

Fam. Ligi i d a e

Ligidium herzegowinense Verhoeff, 1901

Sl 11 - Meča dupka (Vandel, 1967, p.334).

Trogloxène.

Fam. Trich on is c id a e

Sous-fam. T r i ch o n is c in a e

Bureschia bulgarica Verhoeff, 1926

Vr 53 - Beljar (Andreev, 1972, p.178).

Troglobie.

Bulgaronethes haplophthalmoides Vandel, 1967 
Pz 4 - Novata peštera (Vandel, 1967, p.335).

Troglobie.- Espèce endémique de cette grotte.

Balkanoniscus corniculatus Verhoeff, 1926

Razrušcnata peštera (Andreev, 1972, p.179).

Troglobie.

Balkanoniscus minimus Vandel, 1967

Lv 44 - Parnicite (Vandel, 1967, p.335).

Troglobie.- Connu seulement de cette grotte.

Hyloniscus riparius (C.L.Koch, 1838)

Lv 44 - Parnicite (Vandel, 1967, p.335). Mg 2 - Levica vreloto (id.). Pz 4 - Novata peštera (id.). Rz 2 - Mokrata peštera (id.). SI 7 - Niric (id.). Sf 60 - Gornata dupka (id.). Tn 2 - Kalugerova dupka (Andreev, 1972, p.179).

Trogloxène.

Hyloniscus flammula Vandel, 1965

Vd 17 - Elenova dupka (Andreev, 1972, p.179). Vd 18 - Studena (id.).

Troglobie.

Trichoniscus anophthalmus Vandel, 1965

Mg 1 - Mišin kamik (Vandel, 1967, p.336). Vr 42 - Tošova dupka (Andreev, 1972, p.180).

Troglobie.

Trichoniscus anophthalmus intermedius Vandel, 1967

Rs 3 - Tâmnata peštera (Vandel, 1967, p.336).

Troglobie.

Trichoniscus bononiensis Vandel, 1965

Vd 1 - Vârkan (Andreev, 1972, p.180). Vd 8 - Suhi peč (id.). Vd 19 - Redaka 1 (id.). Vd 20 - Mečata dupka (id.). Vd 21 - Prelaz (id.). Vd29 - Jame 3 (id.). Vd 7 - Propast (Andreev, sous presse). Vd 22 - Kračimirskoto vrelo (id.). Vd 44 - Zmijskata propast (id.).

Troglobie.

Trichoniscus semigranulatus Buturovic, 1954

Kl 6 - Meča dupka (Andreev, 1972).

Troglophile.--Connu aussi de Yougoslavie. 
Trichoniscus raitchevi Andreev \& Tabacaru, 1972

Sm 13 - Imamova dupka (Andreev et Tabacaru, 1972).

Troglophile (ou troglobie récent? ). - Pâle, mais pourvu d'ocelles.

Trichoniscus bulgaricus Andreev, 1970

Pv 17 - Julen Ere 2 (Andreev, 1970, p.1135).

Troglobie.

Trichoniscus bureschi Verhoeff, 1926

Lv 50 - ¿ Cerdženica (Andreev, 1972, p.180). Lv 51 - Djado Draganovata peštera (id.). Pv 12 - Cânkaliva dupka (id.). Vr 17 - Ledenika (id.). Vr 22 - Ponora (id.). Dupkata v Glamata (id.).

Troglophile.

Trichoniscus pusillus pusillus Brandt, 1833

Pn 3 (jadis Vr 41) - Temnata dupka (Vandel, 1967, p.336).

Trogloxène.

Trichoniscus tenebrarum Verhoeff, 1926

Tn 3 - Golema Podlisca (Vandel, 1967, p.336).

Troglobie.

Trichoniscus valkanovi Andreev, 1972

Bs 7 - Kirečnicata (Andreev, 1972).

Troglobie.- C'est le premier Isopode troglobie que l'on trouve entre le fleuve Marica et la Mer Noire.

Trichoniscus rhodopienses Vandel, 1965

Sm 15 - Nadarskata peštera (Andreev, 1972, p.180). Sm 16 - Boevskata peštera (id.). Troglobie.-Connu seulement des Rhodopes.

Trichoniscus sp.

Lv 31 - Popskata peštera (Vandel, 1967, p.336).

Sous-fam. H a ploph th a $1 \mathrm{~min}$ a e

Haplophthalmus danicus Budde-Lund, 1885 
Lv 53 - Ljubovnata (Andreev, 1972, p.181).

Trogloxène.- Cette expèce expansive fut trouvée aussi par nous dans un puits à une profondeur de $50 \mathrm{~m}$. près de Général Toševo (Dobrudža)(voir Vandel, 1967). Plusieurs de ces Isopodes rampaient sur un morceau de bois pourri dans une petite caverne naturelle, traversée par le puits.

Cyphoniscellus gueorguievi (Vandel, 1965)

Mg 21 - Srednjata (Andreev, 1972, p.181). Dupkata v Glamata (id.). Vd 14 - Levi Suhi peč (id.). Vd 46 - I'alkovskata peštera (Andreev, sous presse).

Troglobie.

Cyphoniscellus bulgaricus Vandel, 1967

Vr 42 - Tošova dupka (Vandel, 1967, p.337).

Troglobie.

Tricyphoniscus bureschi Verhoeff, 1936

Lv 47 - Bezimenna 22 (Vandel, 1967, p.337). Lv 50 - ¿́erdženica (Andreev, 1972, p.181).

Troglobie.

Beroniscus capreolus Vandel, 1967

Lv 44 - Parnicite (Vandel, 1967, p.337).

Troglobie.

Monocyphoniscus bulgaricus Strouhal, 1939

Kr 3 - Hasarskata peštera (Andreev, 1972, p.181).

Troglophile.

Fam. C y lis t i c i d a e

Cylisticus convexus (de Geer, 1778)

Rz 2 - Mokrata peštera (Vandel, 1967, p.337).

Troglophile.- D'après Vandel (1967), “occasionnellement troglophile”.

Fam. T r a cheli pid a e

Trachelipus rathkei Brandt, 1833

Rz 4 - Kaca peštera (Vandel, 1967, p.338). Vr 68 - Pešketo (Andreev, 1972, p.184).

Trogloxène.- Largement répandu. 
Trachelipus balticus (Verhoeff, 1907)

Sf 12 - Kolibata (à l'entrée de la grotte) (Vandel, 1967, p.338). Pv 1 - Han Maara (id.). Trogloxène.

Tracheoniscus bulgaricus Verhoeff, 1926

Lv 5 - Bankovica (Vandel, 1967, p.339). Lv 16 - Temnata dupka (id.).

Troglophile (?).

Fam. A r m a dillidida e

Armadillidium klugi Brandt, 1833

Lv 36 - Futjovskata peštera (Vandel, 1967, p.340). Sf 36 - Sedmovratica (id.).

Trogloxène.

Armadillidium vulgare (Latreille, 1804)

Lv 5 - Bankovica (Vandel, 1967, p.340).

Trogloxène.

\section{Myriapoda \\ Pauropoda}

Fam. E u r y p a u o p o d i d a e

Eurypauropus cf. cycliger Latzel, 1883

Pv 14 - Ivanova voda (Hazelton, 1970, p.36).

Endogé.- C'est le premier Pauropode signalé des grottes bulgares. Le seul exemplaire capturé (juv.) est mutilé. Il pourrait aussi bien être E.cycliger, E.hastatus Attems ou une expèce nouvelle (Turk, 1970). Les Pauropodes sont en général des animaux rares dans les grottes.

\section{Chilopoda}

Fam. H i m a n t a ri id a e

Thracophilus beroni Matic \& Darabantzu, 1973

Pv 10 - Hralup (Matic et Darabantzu, 1973).

Troglophile (?). 


\section{Fam. Cry p t o p id a e}

Cryptops hortensis Leach, 1814

Lv 2 - Hajduška dupka (Matic, sous presse).

Trogloxène.

\section{Fam. Lit h o bi id a e}

\section{Lithobius agilis C.L.Koch, 1862}

Lv 51 - Djado Draganovata peštera (Matic, sous presse). Pv 1 - Han Maara (id.).

Troglophile.

Lithobius tjasnatensis Matic, 1973

Ja 3 - Tjasnata propast (Matic, sous presse).

Troglobie.

Lithobius popovi Matic, 1973

Lv 37 - Devetaškata peštera (Matic, sous presse).

Troglobie.- Trouvée à $900 \mathrm{~m}$. de l'entrée de la grotte.

Lithobius beshkovi Matic \& Golemansky, 1967

Lv 20 - Rušovata peštera (Matic et Golemansky, 1967, p.127). Lv 28 - Toplja (id.).

Troglophile (? ).-- Pourvue de deux séries d'ocelles, cette espèce n'est connue que des grottes mentionnées, où l'on a aussi trouvé des jeunes et des larves.

\section{Lithobius mutabilis L.Koch, 1862}

Sl 4 - Lednicata (Matic et Golemansky, 1967, p.125).

Trogloxène.

\section{Lithobius lakatnicensis Verhoeff, 1926}

Mg 18 - Grebenjo (Matic, sous presse). Sm 6 - Čelečkata pestera (id.). Sm 23 - Rizovica (id.). Vr 42 - Tošova dupka (id.).

Troglobie.

\section{Lithobius piceus L.Koch, 1865}

Ja 8 - Kesadžijca (Matic, sous presse)(avec “? ”).

Trogloxène. 
Lithobius erythrocephalus C.L.Koch, 1847

Sf 74 - Padež (Matic, sous presse, avec “? ").

Trogloxène.

Monotarsobius crassipes (L.Koch, 1862)

Pv 4 - Topčika (Matic, sous presse). Pv 8 - Jamata (id.).

Trogloxène.

Monotarsobius bifidus Matic, 1972

Bs 6 - Izvornata peštera (Matic, sous presse).

Troglobie (? ).-- Ce Chilopode n'est pas rare dans la grotte. Il a 3-4 ocelles.

\section{Fam. S c u t ig e rid a e}

\section{Scutigera coleoptrata Linnaeus, 1758}

Pv 13 - Fidjafkinata dupka (Matic, sous presse). Vd 37 - Magura 2 (id.).

Trogloxène.

\section{Diplopoda}

Fam. Trach y s pha erida e

\section{Trachysphaera costata (Waga, 1857)}

Lv 20 - Rušovata peštera (Strasser, 1969, p.138). Mg 15 - Ajdučkata peštera (id.). Šn 4 Dipsiskuja (id.). Vr 37 - Govedarskata dupka (id.). Pn 2 - Hajduškata peštera (Strasser, in lit.). Pn 4 - Sedlarkata (id.). Pv 17 - Julen ere 2 (id.). Vr 34 - Dupna mogila (id.).

Troglophile.

Trachysphaera orghidani (Tabacaru, 1958)

Sf 26 - Svârdelo (Strasser, 1969, p.131). Vd 16 - Tâmna dupka (id.). Sf 34 - Kozarskata peštera (Strasser, $\mathrm{n}$ lit.).

Troglobie.

$$
\text { Fam. P oly d e s m i d a e }
$$

Polydesmus (P.) complanatus illyricus Verhoeff, 1893

Pv 17 - Julen ere 2 (Strasser, in lit.).

Trogloxène. 
Polydesmus (Nomarchus) denticulatus C.L.Koch, 1847

Tg 2 - Prolazkata peštera (Strasser, 1969, p.138).

Trogloxène.- Dans le travail de Strasser il y a eu une substitution typographique des noms "Polydesmus denticulatus" et "P.denticulatus beroni".

Polydesmus (N.) denticulatus beroni Strasser, 1962

Sl 20 - Mâglivata peštera (Strasser, 1969, p.138).

Troglophile (?).

Polydesmus (N.) renschi Schubart, 1934

Mg 16 - Dupkata (Strasser, 1969, p.139). Mg 23 - Micina dupka (id.). Vd 4 - Neprivetlivata (Strasser, in lit.).

Trogloxène.

Polydesmus (N.) mediterraneus valachicus Tabacaru et Negrea, 1961

Lv 44 - Parnicite (Strasser, 1969, p.139).

Trogloxène.

Brachydesmus (B.) dadayi Verhoeff

Vd 16 - Tâmna dupka (Strasser, in lit.).

Trogloxène.

Brachydesmus (B.) dadayi brusenicus Gulička, 1967

Vd 4 - Neprivetlivata (Strasser, in lit.).

Trogloxène.

Brachydesmus (B.) herzegowinensis confinis Strasser, 1938

Sf 8 - Temnata dupka (Strasser, 1969, p.141).

Troglophile (?).-- L'espèce B.herzegowinensis est connue de Yougoslavie (B.h. herzegowinensis et B.h.septentrionalis) et de Bulgarie (B.h.confinis). D'après Strasser (1969), "B.h.herzegowinensis wurde sowohl im Freien wie auch in Höhlen gefunden; von den beiden anderen Rassen sind nur Funde aus Höhlen bekannt". Dans la grotte Temnata dupka ces Diplopodes vivent à $450 \mathrm{~m}$ à l'intérieur de la grotte.

Brachydesmus (B.) cristofer Strasser, 1966

Kl 4 - Jamkata (Strasser, 1969, p.142). Pk 1 - Duhlata (id.). 
Troglophile (? ).- Dans les galeries humides au lieu-dit Urvič (20 km S de Sofia), qui sont creusées artificiellement dans un terrain non-karstique, ces Diplopodes se trouvent en quantité en automne. On les voit le plus souvent rampant au plafond des galeries, plus rarement sur le sol.

\section{Fam. Antrole ucosomatida e}

Bulgarosoma meridionale Tabacaru, 1967

Sm 13 - Imamova dupka (Tabacaru, 1967, p.22).

Troglobie.

Bulgarosoma tridentifer (Gulička, 1967)

Sm 6 - Člečkata peštera (Gulička, 1967, p.2).

Troglobie.- Cette espèce a été décrite comme type du genre Troglodicus Gulička, 1967, mais Tabacaru (1970, Livre de Centenaire de Racovitza p.427) l'a mise dans le genre Bulgarosoma.

Stygiosoma beroni Gulička, 1967

Bl 1 - Manailovata peštera (Gulička, 1967a, p.2).

Troglobie.

Antrodicus falcatus Gulička, 1967

Sl 6 - Kipilovskata peštera (nommée aussi "Šaplâka" ou "Šaplâška", mais non pas "Šapaška") (Gulička, 1967a, p.3).

Troglophile (?).

Fam. C h or d e u m id a e

Microchordeuma sp.

Tn 2 - Kalugerova dupka (Strasser, 1969, p.145).

Fam. Ly s i o p e t alid a e

Balkanopetalum armatum Verhoeff, 1926

Vr 64 - Šš̌manovec (Strasser, 1969, p.145). Sf 76 - Paraklisa (Strasser, in lit.).

Troglophile.

Balkanopetalum rhodopinum Verhoeff, 1937

Pv 8 - Jamata (Strasser, 1969, p.145). Pv 10 - Hralup (id.). Sm 30 - Garvanjovica (id.).

Troglophile. 
Balkanopetalum beshkovi Strasser, in lit.

Pv 4 - Topčika (Strasser, in lit.).

Troglophile.

$$
\text { Fam. J u } 1 \text { i d a e }
$$

Chromatoiulus rhodopinus Verhoeff, 1928

Sm 19 - Dolna Karanska dupka (Strasser, 1969, p.159).

Trogloxène.

Chromatoiulus beroni Strasser, in lit.

Sm 32 - Djavolsk oto gârlo (Strasser, in lit.).

Trogloxène.

Nopoiulus venustus (Meinert, 1868)

$(=N$.pulchellus Leach $=N$. armatus Nemec

Mg 3 - Vreloto (Strasser, 1969, p.165). Pn 4 - Sedlarkata (Strasser, in lit.).

Troglophile.

Apfelbeckiella trnovensis (Verhoeff, 1928)

Šn 1 - Katakombite (Strasser, 1969, p.164).

Troglobie (?).

Typhloiulus georgievi Strasser, 1962

Lv 31 - Popskata peštera (Strasser, 1969, p.152).

Troglobie (?).

Typhloiulus kotelensis Jawlowski, 1938

Sl 20 - Mâglivata peštera (Strasser, 1969, p.151).

Troglophile ou trogloxène.- En étudiant cet animal, Mr Strasser admet que "liefern die Antennen die Bestätigung, dass T. kotelensis kein Höhlentier ist".

Typhloiulus bureschi Verhoeff, 1926

Sf 28 - Mečata dupka (Strasser, 1962, Atti Mus.Civ.St.Nat.Trieste, p.53, sub "Yzdremetzka I bei Zeleu sic", réf.omise dans notre travail de 1967. Vr 44 - Popovata peštera (Popov, 1969, p.39). Vr 58 - Moravata (id., p.37). Lv 47 - Bezimenna 22 (Strasser, 1969, p.150). Lv 70 Sopotskata peštera (Strasser, in lit.). Mg 19 - Nikolova jama (id.). Sf 74 - Padež (id.). Sf 76 Paraklisa (id.). Vr 42 - Tošova dupka (id.). Vr 22 - Ponora (id.).

Troglobie.- Cette espèce paraît localisée dans la chaîne de Stara Planina au Sud-Est 
de la rivière Ogosta. Il faut noter, que Strasser (1969) écrit: "Sicher nicht zu T.bureschi gehört die von mir 1966 angeführte Larve aus der Höhle Vodni peč (Vd 15) bei Dolni Lom".

Typhloiulus longipes Strasser, 1973

Vr 53 - Beljar (Strasser, in lit.).

Troglobie.

Typhloiulus strictus Latzel, 1882

Vd 1 - Varkan (Strasser, in lit.). Vd 33 - Koritska glama (id.).

Troglophile.- Cette espèce était connue jusqu'à présent du Banat et de la Serbie du Nord-Est.

Typhloiulus staregai Strasser, 1973

Vd 21 - Prelaz (Strasser, in lit.).

Troglobie.

Serboiulus spelaeophilus Gulička, 1967

Mg 13 - Sokjovec (Strasser, 1969, p.154). Mg 14 - Mramornata peštera (id.). Vd 16 - Tâmna dupka (id.). Vd 15 - Vodni peč (Gulička, 1967a, p.4). Vd 10 - Tâmni peč (Strasser, in lit.). Vd 19 - Redaka 1 (id.). Vd 29 - Jame 3 (id.). Vd 34 - Redaka 2 (id.). Vd 38 - Stanišina dupka (id.). Vd 41 - Medžak dupka (id.). Vd 44 - Zmijskata propast (id.). Vd 46 - Falkovskata peštera (id.). Vd 50 - Zankovska peštera (id.).

Troglobie.- Espèce caractéristique de la Stara planina de l'Ouest. Comme nous a fait aimablement savoir Mr Strasser, il a établi la synonymie suivante: Serboiulus (Balkaniulus) spelaeophilus Gulička, $1967=$ S. popovi Strasser, 1969.

\section{Arachnida \\ Araneida}

Fam. Ph ol c i d a e

Hoplopholcus forscali (Thorell, 1871)

Kl 2 - Boboševskata peštera (Deltshev, 1972, p.172). Lv 17 - Dolnata peštera (id.). Lv 34 Vodopada (id.). Lv 55 - Peštera 4 (id.). Vr 3 - Studenata dupka (id.). Vr 42 - Tošova dupka (id.). Vr 54 - Briša (id.). Mg 22 - Gornjata, 16.X.1969, P.Beron leg. (Hr.Delčev det.).

Trogloxène régulier.

Pholcus opilionoides Schrank, 1783

Vd 31 - Dragaličeva dupka (Deltshev, 1972, p.172).

Trogloxène régulier. 
Fam. N e s t ic id a e

\section{Nesticus eremita Simon, 1879}

Šn 2 - Arkovna (Deltshev, 1972, p.172).

Troglophile.

Nesticus cellulanus Clerck, 1757

(Hr.Delčev, P.Beron et al. leg., Hr.Delčev det.)

Bs 4 - Jamata, 9.VIII.1970. Bs 5 - Ezeroto, 7.V.1963. Ja 5 - Drânci dupka, 6.VIII,1970. Ja 9 - Sirokija kajrjak, le 6.VIII.1970. K1 6 - Meča dupka, 25.II.1969. Lv 4 - Svirčovica, 16.VIII.1966. Lv 5 - Bankovica, 4.IX.1965. Lv 10 - Zadânenka, 15.VII.1963. Lv 34 Vodopada, 13.VIII.1968. Lv 35 - Uruška maara, 12.VII.1965. Lv 44 - Parnicite, 7.I.1963. Lv 53 - Ljubovnata, 25.VIII.1966. Lv 54 - Zalâmska dupka, 2.IX.1965. Lv 58 - Skravenika, 18.VIII.1966. Lv 69 - Enčova dupka, 18.VIII.1966. Lv 79 - Imaneto, 25.VIII.1966. Lv 7 Temnata dupka, 23.VIII.1963. Lv 80 - Propast 112, 24.III.1968. Mg 11 - Mitrevska dupka, 26.I.1971. Mg 19 - Nikolova jama, 24.XII.1969. Pk 2 - Zivata voda, 24.X.1963. Pn 6 Gornoto ezero, 20.X.1968. RZ 2 - Mokrata peštera, 8.V.1966. Rz 4 - Kaca peštera, 8.V.1966. Sl 16 - Stârganakovoto, 7.VIII.1962. Sl 21 - Nehrov trap, 7.X.1966. Sm 13 - Imamova dupka, 26.VI.1966. Sm 18 - Sančova dupka, 27.VI.1966. Sm 32 - Djavolskoto gârlo, 30.VI.1966. Sn 5 - Zâlâd, 9.IV.1967. Sn 8 - Lozevskata peštera, 22.IX.1968. Tn 10 Draganovskata peštera, 8.X.1962. Vd 1 - Vârkan, 20.X.1969. Vd 10 - Tâmni peč, 30.10.1967. Vd 17 - Elenova dupka, 19.X.1969. Vd 18 - Studena, 28.X.1969. Vd 32 - Djado Stanov stol, 12.X.1969. Vd 35 - Carevica, 22.X.1969. Vr 2 - Drašanskata peštera, 20.X.1968. Vr $17-$ Ledenika, 2.IX.1965. Vr 22 - Ponora, 18.VIII.1965. Vr 28 - Tigančeto, 16.VIII.1963. Vr 30 - Mladenovata peštera, 17.VIII.1963. Vr 42 - Tošova dupka, 12.XII.1965. Vr 49 - Capkula, 19.XII.1965. Vr 51 - Vodnata peštera, 1.V.1966. Vr 55 - Barkite 1, 11.X.1968. Vr 44 Popovata peštera, (Popov, 1969, p.39).

Troglophile.- Espèce largement répandue en Bulgarie.

Fam. Li n y p h i id a e

\section{Centromerus bulgarianus Drensky, 1931}

Vr 52 - Sokolskata dupka (Deltshev, 1972, p.172). Vr 53 - Beljar (id.).

Troglobie.- En dehors de ces deux grottes, C.bulgarianus n'était connue que de la grotte Ražiška dupka près de Lakatnik. C'est l'unique Araignée troglobie que l'on connaisse en Bulgarie.

\section{Centromerus jacksoni Denis, 1952}

Lv 57 - Gurlieva dupka (Deltshev, 1972 p.172). Sf 3 - Dinevata pešt (id.). Sm 16 - Boevskata peštera (id.). Vd 20 - Mečata dupka (id.).

Troglophile.

\section{Centromerus lakatnikensis Drensky, 1931}

Sf 59 - Radjova propast (Deltshev, sous presse).

Troglophile. 
Lepthyphantes tenuis (Blackwall, 1852)

Lv 53 - Ljubovnata (Deltshev, 1972 p.172).

Trogloxène.

Lepthyphantes zimmermani Bertkau, 1890

Pz 7 - Dupčeto (Delčev, sous presse).

Trogloxène.

Lepthyphantes rhodopensis (Drenksky, 1931)

Sm 26 - Rizovica (Deltshev, sous presse). Bl 5 - Bânderica (id.).

Troglophile.- Paraît propre aux Rhodopes, Rila et Pirin.

Lepthyphantes balcanica Drensky, 1931

Lv 52 - Sinjoto kolelo (Deltshev, sous presse).

Troglophile.- C'est la troisième localité de cette espèce (toutes les trois se trouvent dans la région du Prébalkan Central).

Lepthyphantes leprosus Ohlert, 1865

Lv 2 - Hajduška dupka (Deltshev, sous presse). Lv 12 - Ledenicata (id.). Lv 17 - Dolnata peštera (id.). Pv 10 - Hralup (id.). Pv 15 - Hajduškata dupka (id.). Rz 3 - Kulina dupǩa (id.). Rz 4 - Kaca peštera (id.). Rs 4 - Propastta (id.). Sl 21 - Nehrov trap (id.). Sn 1 Katakombite (id.). Vd 10 - Tâmni peč (id.). Vd 11 - Golemi peč (id.). Vd 22 - Kračimirsk oto vrelo (id.). Vd 27 - Jame 1 (id.). Vr 1 - Prilepnata peštera (id.). Vr 16 - Reznjovete (id.). Vr 56 - Propast 2 (id.). Ja 2 - Stara Sveta Trojca, 5.VIII.1970, P.Beron et V.Beškov leg. (Hr.Delčev det.).

Troglophile.

Lepthyphantes sofianus (Drensky, 1931) (syn. Lepthyphantes tranteevi Miller, 1958)

Mg 12 - Grimnena dupka (Deltshev, sous presse). Sf 74 - Padež (id.). Vr 17 - Ledenika (id.). Vr 29 - Sipo (id.). Vr 32 - Zmejova dupka II (id.). Vr 55 - Barkite 1 (id.).

Troglophile.

Lepthyphantes insignis Cambridge, 1913

Pv 13 - Fidjafkinata dupka (Deltshev, 1972, p.172). Rs 1 - Orlova čuka (id.). Ja 8 Kesadžijca, le 5.VIII.1970, P.Beron et V.Bešk ov leg. (Hr.Delčev det.).

Troglophile.

Lepthyphantes spelaeorum Kulczinski, 1914

(syn. Troglohyphantes [sic] trnovensis Drensky, 1931) 
Kl 3 - Ilijskata peštera (Deltshev, 1972, p.172). Lv 5 - Bankovica (id.). Lv 48 - Propast 30 (id.). Lv 49 - Dâlbokata propast (id.). Lv 53 - Ljubovnata (id.). Lv 64 - Malkata Cervenska jama (id.). Mg 16 - Dupkata (id.). Vd 8 - Suhi peč (id.). Vd 10 - Tâmni peč (id.). Vd 20 Meča dupka (id.). Vd 29 - Jame 3 (id.). Vd 30 - Peina dupka (id.).

Troglophile.

Lepthyphantes centromeroides Kulczinski, 1914

(syn. Troglohyphantes bureschi Drensky, 1931

Lept.bureschi bureschi (Dren.)(Dum. \& Georg., 1970)

Pv 14 - Ivanova voda (Hazelton, 1970, p.36). Vr 17 - Ledenika (Dumitresco \& Georgesco, 1970, p.325). Sm 18 - Dolna Karanska dupka (id.). Sm 19 - Sančova dupka (id.). Lv 49 Dâlbokata peštera (Deltshev, sous presse). Sf 25 - Javoreckata peštera (id.). Sf 68 - Svinaka (id.). Sm 16 - Boevskata peštera (id.). Sm 21 - Handaka (id.). Vd 2 - Magura (id.).

Troglophile.

Antrohyphantes rodopicus Dumitresco, 1971

Sm 22 - Meča dupka (Dumitresco, 1971, p.167).

Troglophile.

Porrhomma convexum (Westring, 1861)

= P.rosenhaueri et P.errans sensu Drensky, 1931

=P.profundum sensu Guéorguiev \& Beron, 1962)

Pv 14 - Ivanova voda (Hazelton, 1970, p.36). Bs 4 - Jamata, le 9.VIII.1970, P. Beron et V.Beškov leg. (Hr.Deľ̌ev det.). Ja 1 - Bozkite, 4.VIII.1970 (id.). Ja 5 - Drânči dupka, 6.VIII.1970 (id.). Ja 12 - Leleška dupka 1, 8.VIII.1970 (id.). Ja 15 - Jamata, 9.VIII.1970 (id.). Mg 17 - Drânkalna dupka, 14.VI.1969 (id.). Pv 1 - Han maara, 7.IV.1970, P.Beron leg. (Hr. Delčev det.). Pv 9 - Erkjuprija, 18.III.1968 (id.). Sf 75 - Blagova dupka 1, 10.VIII.1968 (id.).

Troglophile.- Très répandu dans les grottes bulgares. Il paraît que toutes les Porrhomma, publiées de Bulgarie, appartiennent à cette espèce.

Porrhomma sp.

Vr 58 - Moravata (Popov, 1969, p.37). Pv 14 - Ivanova voda (Hazelton, 1970, p.34).

Fam. Le p t o n e t id a e

Protoleptoneta bulgarica Deltshev, 1972

Lv 47 - Bezimenna 22 (Deltshev, 1972). Mg 18 - Grebenjo (id.). Vr 34 - Dupna mogila (id.). Troglophile.- C'est le premier représentant de la famille des Leptonetidae en Bulgarie. L'espèce paraît cantonnée dans le Prébalkan Occidental. 
Fam. M i c ry phantid a e

Diplocephalus cristatus (Blackwall, 1833)

Lv 4 - Svirčovica (Hazelton, 1970, p.33).

Trogloxène.

Fam. A r a n e i d a e

Araneus bituberculatus Walkenaer, 1802

Vd 22 - Kračimirsk oto vrelo (Deltshev, 1972 p.173).

Trogloxène.

Meta segmentata (Clerck, 1757)

Sl 21 - Nehrov trap (Deltshev, 1970, p.91). Vr 46 - Dupkata (id.).

Trogloxène.

\section{Meta merianae (Scopoli, 1763)}

Gb 7 - Izvora (Delčev, 1970, p.91). Lv 2 - Hajduška dupka (id.). Lv 35 - Uruška maara (id.). Lv 58 - Skravenika (id.). Mg 2 - Levica vreloto (id.). Pn 6 - Gornoto ezero (id.). Pv 7 Gargina dupka (id.). Sf 7 - Bučinska pešt (id.). Sf 24 - Meča dupka (id.). Sf 67 - Jamata (id.). Sn 1 - Katakombite (id.). Vd 22 - Kračimirskoto vrelo (id.). Vr 42 - Tošova dupka (id.). Vr 44 - Popovata peštera (id.). Vr 45 - Meča dupka (id.). Ja 13 - Leleška dupka 2, 8.VIII.1970, P.Beron et V.Beškov leg. (Hr.Delčev det.).

Troglophile.

\section{Meta menardi (Latreille, 1804)}

Gb 7 - Izvora (Deltshev, 1970, p.90). Gb 8 - Ivankinata peštera (id.). Kl 3 - Ilijskata peštera (id.). K1 7 - Vâlči dol (id.). Kr 1 - Tilki ini (id.). Lv 4 - Svirčovica (id.). Lv 54 - Zalâmska dupka (id.). Lv 56 - Propast 65 (id.). Lv 58 - Skravenika (id.). Lv 60 - Bojkina peštera (id.). Lv 61 - Propast 71 (id.). Lv 62 - Dolnata Cervenska dupka (id.). Lv 63 - Gornata Cervenska dupka (id.). Lv 65 - Meča dupka (id.). Lv 66 - Svinštica (id.). Mg 1 -- Mišin kamik (id.). Mg 7 - Jamata (id.). Mg 8 - Peč (id.). Mg 16 - Dupkata (id.). Pk 4 - Filiposvskata peštera (id.). Pk 7 - Bezimenna peštera (id.). Pk 8 - Cerna padina (id.). Pv 8 - Jamata (id.). Pv 10 - Hralup (id.). Rz 2 - Mokrata peštera (id.). Rz 4 - Kaca peštera (id.). Sl 8 - Orlovata peštera (id.). Sl 11 - Mečata dupka (id.). Sl 17 - Dimova dupka (id.). Sm 10 - Vaklite dupki (id.). Sm 13 Imamova dupka (id.). Sm 17 - Borovskata peštera (id.). Sm 18 - Sančova dupka (id.). Sm 20 - Gorna Karanska dupka (id.). Sm 22 - Meča dupka (id.). Sm 24 - Ramadanovata dupka (id.). Sm 25 - Malkata peštera (id.). Sf 3 - Dinevata pešt (id.). Sf 9 - Peruna dupka (id.). Sf 24 Meča dupka (id.). Sf 33 - Svinskata dupka (id.). Sf 38 - Kitova kukla (id.). Sf 44 - Suhata jama (id.). Sf 47 - Vodnata pešt (id.). Sf 69 - Hajduškata dupka (id.). Sf 70 - Meča dupka (id.). Sf 71 - Pepina dupka (id.). Sf 72 - Zdraveška dupka (id.).

Petite grotte près de Razboïski monastir (id.).

Petite grotte près du v.Lopušna, au lieu-dit Mogilo.

Petit gouffre près du v.Lopušna, au lieu-dit Mogilo. 
Sz 3 - Toplata dupka (id.). Tn 10 - Draganovskata peštera (id.). Tn 11 - Jamata (id.). Šn 1 Katakombite (id.). Sn 2 - Arkovna (id.). Sn 5 - Zâlâd (id.). Vd 22 - Krarimirskoto Vrelo (id.). Vd 23 - Studeni vrâh (id.). Vd 24 - Spuštenica (id.). Vr 16 - Reznjovete (id.). Vr 17 Ledenika (id.). Vr 23 - Golemata jama (id.). Vr 25 - Zmejova dupka (id.). Vr 42 - Tošova dupka (id.). Vr 48 - Ezeroto (id.). Vr 49 - Capkula (id.). Vr 50 - Propast 8 (id.). Vr 51 Vodnata dupka (id.). Vr 52 - Sokolskata dupka (id.). Bezimenna près de Šumen - grotte non localisée (idem).

Troglophile.

\section{Meta bourneti Simon, 1922}

Ja 16 - Ostâr kamâk: (Delčev, 1970, p.89). Ja 2 - Stara Sveta Trojca, 5.VIII.1970, P. Beron et V. Beškov leg. (Hr.IJelčev det.). Ja 3 - Tjasnata propast, 5.VIII.1970 (id.). Ja 4 - Drânči dupka, 4.VIII.1970 (id.). Ja 6 - Kara burnu, 6.VIII.1970 (id.). Ja 7 - Suhata dupka, 5.VIII.1970 (id.).

Troglophile.- En Bulgarie cette espèce paraît localisée dans les grottes de Strandža, de Sakar et du littoral de la Mer Noire.

\section{Fam. A g e le n i d a e}

Tegenaria domestica (Clerck, 1757)

Kl 2 - Boboševskata peštera (Deltshev, 1972, p.173). Kl 5 - Kacite (id.). Rs 5 - Dil Nikolca (id.). Rz 2 - Mokrata peštera (id.). Sf 73 - Vâlči kamâk (id.). Vd 11 - Golemi peč (id.).

Trogloxène régulier.

Tegenaria ferruginca (Panzer, 1804)

Sm 20 - Gorna Karanska dupka (Deltshev, 1972, p.173). Vd 22 - Kračimirskoto vrelo (id.). Vr 54 - Briša (id.).

Trogloxène régulier.

Tegenaria silvestris L.Koch, 1873

Lv 4 - Svirčovica (Deltshev, 1972, p.173). Pn 6 - Gornoto ezero (id.). Pv 1 - Han maara (id.). Sf 7 - Bučinskata pešt (id.). Sm 16 - Boevskata peštera (id.). Sn 3 - Peštera 5 (id.). Tn 10 Draganovskata peštera (id.). Vr 3 - Studenata dupka (id.). Vr 17 - Ledenika (id.). Vr 52 Sokolskata dupka (id.).

Trogloxène régulier.

\section{Fam. L y c o s i d a e}

\section{Pardosa hortensis (Thorell, 1872)}

Petit gouffre près du v.Lopušna (Deltshev, 1972, p.173).

Trogloxène occasionnel. 
Amaurobius pallidus L.Koch, 1868

Petit gouffre près du v.Lopušna (Deltshev, 1972, p.174).

Trogloxène occasionnel.

Amaurobius claustrarius (Hahn, 1831) et Amaurobius claustrarius balcanicus Drensky, 1940

Sm 13 - Imamova dupka (Dumitrescu et Orghidan, 1969, p.61).

Trogloxène.

Ac aromorpha

Nous pensons que pour les buts de cette publication il n'est pas nécessaire d'énumérer plus loin toutes les grottes où des Acariens parasites ont été trouvés. Nous n'énumérons ici que les Acariens (resp. leurs stades) qui vivent librement dans les grottes. Les stades parasitaires ne sont mentionnés que d'une manière générale. Ils sont ordinairement des parasites des Chauves-souris.

\section{A c a riformes \\ Fam. A c a rid a e}

Schwiebea cavernicola (Vitzthum, 1932)

Lv 5 - Bankovica (Turk, 1970, p.40).

Trogloxène.

Fam. S a r c p tid a e

Nycteridocoptes poppei (Oudemans, 1897) - Beron, 1970, p.146.

N.eyndhoveni Fain, 1959 - Beron, 1970, p.147.

Fam. La b i d o c a r p i d a e

Alabidocarpus calcaratus Lawrence, 1953 - Beron, 1970, p.148.

Fam. Rh a gi d i i d a e

Rhagidia spelaea (Wankel, 1861)

Lv 4 - Svirčovica (Turk, 1970, p.40). Lv 5 - Bankovica (id.). Pv 13 - Ivanova voda (id.). Endogé(? ) - Trouvé dans les grottes en Europe.

Rhagidia strouhali Willmann, 1953 
Lv 5 - Bankovica (Turk, 1970, p.40).

Endogé.

Rhagidia wolmsdorfensis Willmann, 1936

Pv 13 - Ivanova voda (Turk, 1970, p.40).

Endogé.- Dans les grottes et sous pierres.

Fam. Er e y n e t i d a e

Ereynetes simplex Willmann, 1936

Lv 5 - Bankovica (Turk, 1970, p.40).

Trogloxène.-- Connu d'Autriche (mine de graphite). D'après Turk (loc.cit.), "The single example from the Bankovitza cave perhaps represents a new sub-species".

Fam. C u n a id a e

Cunaxoides croceus (Koch, 1838)

Pk 1 - Duhlata (Turk, 1970, p.40).

Trogloxène.

Fam. B d e 11 i d a e

Odontoscirus iota Sosnina et al., 1965

Lv 5 - Bankovica (Turk, 1970, p.40). Pk 1 - Duhlata (id.).

Trogloxène.

Fam. Tr o m b i c ulid a e

Neotrombicula autumnalis (Shaw, 1790)

Lv 4 - Svirčovica (Turk, 1970, p.40). Pk 1 - Duchlata (id.).

Parasite.

Neotrombicula (N.) rhinolophi Kolebinova, 1968 - Kolebinova, 1968, p.383

Fam. M y o b i i d a e

Neomyobia chiropteralis (Michael, 1884) - Beron, 1968, p.158; 1970, p.145.

N.rollinati (Poppe, 1908) - Beron, 1968, p.158; 1970, p.145. L'espèce communiquée par Kolebinova (1967) sub “'N.rollinati” appartient en effet à une autre espèce.

Acanthophthirius klapaleki (Dusbábek, 1963) - Beron, 1970, p.144.

A. emarginatus (Dusbábek, 1963) - id. 
A.etheldredae Perkins, 1925 - Beron, 1968, p.158, sub "Neomyobia poppei". A.myoti (Dusbábek, 1963) - Kolebinova, 1967, p.73; Beron, 1970, p.144.

Calcarmyobia rhinolophia (Radford, 1940) - Beron, 1970, p.145.

Pteracarus submedianus Dusbábek, 1963 - Beron, 1968, p.157.

P.pipistrellius (Radford, 1938) - id.

Parasitiformes

Fam. M a c r on y s sid a e

Ichoronyssus scutatus (Kolenati, 1856) - Beron, 1968, p.159.

Macronyssus cyclaspis (Oudemans, 1906) - id.

M. ellipticus (Kolenati, 1856) - id.

M.flavus (Kolenati, 1856) - id.

M.granulosus (Kolenati, 1856) - id .

M.rhinolophi (Oudemans, 1902) - id.

Steatonyssus spinosus Willmann, 1936 - id. p.160.

S.periblepharus Kolenati, 1858 - id.

Fam. S p in t u r n i c i d a e

Eyndhovenia euryalis (Canestrini, 1884) - Beron, 1968, p.158; 1970, p.146.

Paraperiglischrus rhinolophinus (C.L.Koch, 1841) - Beron, 1968, p.158.

Spinturnix mystacinus (Kolenati, 1857) - id.

S.myoti (Kolenati, 1856) - id.

Insecta

Colle mbola

Fam. On y c hiuridae

Onychiurus tuberculatus (Moniez, 1890)

Lv 59 - Goljamata jama, 24.XI.1968, P.Beron leg. (M.Gruia det.).

Troglophile.

Onychiurus ghidinii Denis, 1938

Sf 70 - Meča dupka, 24.IX.1961 (M.Gruia det.).

Troglophile.- Deuxième localité en Bulgarie.

Onychiurus subgranulosus Gama

Vr 47 - Lednicata, 10.X.1968, P.Beron leg. (M.Gruia det.).

Troglophile.- Connu de Yougoslavie et de Bulgarie. 
Onychiurus fimetarius (groupe)

Pk 1 - Duhlata (Hazelton, 1970, p.33).

Fam. S m in th u rid a e

Arrhopalites pygmaeus (Wankel, 1860)

Lv 4 - Svirčovica (Hazelton, 1970, p.33). Pk 1 - Duhlata (id. p.34).

Troglophile.- Largement répandue dans les grottes européennes.

Fam. En t o m obry i d a e

Orchesella cf. alticola Uzel, 1890

Lv 4 - Svirčovica (Hazielton, 1970, p.33).

Orchesella sp.

Pv 14 - Ivanova voda (id., p.36).

Heteromurus nitidus (Tampleton, 1835)

Lv 5 - Bankovica (Hazelton, 1970, p.33). Vd 8 - Suhi peč, 17.X.1971, P.Beron leg. (M.Gruia det.). Vd 7 - Propast, 19.X.1971, id. Vd 46 - Falkovskata peštera, 18.X.1971, id.

Troglophile.- Espèce commune en Bulgarie. En dépit de l'affirmation de Turk (1970, p.41), cette espèce est mentionnée dans notre Essai (1962, p.320-321 et 1967, p.171) de 13 grottes bulgares.

Heteromurus nitidus quadriocellatus Ksenemann, 1935

Vd 42 - Golema dupka, 22.X.1971, P.Beron leg. (M.Gruia det.) Vd 50 - Zankovska peštera, 21.X.1971 (idem).

Troglophile.

Pseudosinella duodecimocellata Handschin, 1928

Ve 47 - Lednicata, 10.X.1968, P.Beron leg. (M.Gruia det.).

Troglobie.

Pseudosinella cf fallıx Börner, 1903

Lv 5 - Bankovica (Hazelton, 1970, p.34).

Pseudosinella sp.

Lv 64 - Malkata jama, 24.XI.1968, P.Beron leg. (M.Gruia det.).

Lepidocyrtus sp.

Pv 14 - Ivanova voda (Hazelton, 1970, p.36). 
Lv 5 - Bankovica (Hazelton, 1970, p.34). Pv 14 - Ivanova voda (id. p.36).

Fam. I s o t o m i d a e

Isotomiella minor (Schäffer, 1896)

Pv 14 - Ivanova voda (Hazelton, 1970, p.36).

Trogloxène.

Folsomia candida (Willem, 1902)

Vr 51 - Ohodenskata peštera, 18.VI.1971, P.Beron leg. (M.Gruia det.).

Troglophile.-- Largement répandu en Europe.

Folsomia sp.

Lv 5 - Bankovica (Hazelton, 1970, p.33).

Fam. T o m o c e rid a e

Tomocerus minor Lubbock, 1862

Lv 59 - Goljamata jama, 24.XI.1968, P.Beron leg. (M.Gruia det.).

Troglophile.

Tomocerus cf. unidentatus Börner, 1901

Pv 14 - Ivanova voda (Hazelton, 1970, p.36).

Fam. H y p og a s r u ridae

Ceratophysella armata (Nicolet, 1841)

Sf 41 - Čavkite, 14.VII.1933, (J.-M.Thibaud det.). Vr 40 - Kalnata dupka, 27.IX.1968, P.Beron leg. (M.Gruia det.). Vr 42 - Tošova dupka, 17.II.1968, P.Beron leg. (M.Gruia det.).

Trogloxène.

Hypogastrura cf. tullbergi (Schäffer, 1900)

Lv 5 - Bankovica (Hazelton, 1970, p.33).

Pseudacherontides spelaea (Ionesco, 1923)

Pz 4 - Novata peštera, 15.X.1932 (J.-M.Thibaud det.).

Troglobie.- Connue de Roumanie. Espèce nouvelle pour la faune bulgare. 
Fam. N e a n r i d a e

Lathriopyga phlegraea (Caroli, 1912)

Lv 59 - Goljamata jama, 24.XI.1968, P.Beron leg. (M.Gruia det.).

Trogloxène.

Bilobella digitata Cassagnau, 1968

Sl 9 - Drjanovskata peštera (Cassagnau, 1968, p.302).

Endogé.- L'espèce est décrite de Grèce (lavages de terre) et de la susdite grotte bulgare.

$$
\text { Fam. O n c o p o d u ri d a e }
$$

Oncopodura sp.

Lv 4 - Svirčovica (Hazelton, 1970, p.33). Lv 5 - Bankovica (idem, p.34).

\section{Trirhacus sp.}

Hom optera

Fam. C i x i i d a e

Sm 30 - Garvanjovica, 3.IV.1967, P.Beron leg. (I.Dlabola det.). Sm 25 - Malkata peštera, 2.IV.1967 (idem).

Trogloxène.- Les larves de ces Cixiides ont été trouvées rampant sur l'argile humide, bien à l'intérieur des grottes. C'est rarement qu'on trouve des Cicades dans les grottes européennes, où les représentants aveugles de ce groupe sont complètement inconnus. Ces derniers existent, par contre, à Madagascar.

$$
\text { Orthoptera }
$$

Fam. R a phid oph orid a e

Troglophilus sp.

Sm 25 - Malkata peštera, (P.Beron leg.et det.). Sm 26 - Červena dupka (idem).

Des Orthoptères du genre Troglophilus ont été récoltés dans plusieurs grottes le long de la frontière yougoslavo-bulgare et dans les Rhodopes, mais le matériel n'est pas encore déterminé. Les Troglophilus des deux susdites grottes ont été récoltés le 2.IV.1967 et publiées dans une note anonyme (sub "sauterelles cavernicoles") dans la revue "Rodopski pesternjak", IV, 34, p.44-45).

$$
\begin{gathered}
\text { Coleoptera } \\
\text { Fam. Carabidae }
\end{gathered}
$$

Duvalius (Duvaliotes) beshkovi Coiffait, 1970 
K1 6 - Meča dupka (Coiffait, 1970, p.722; il faut lire la localité: Grotte Meča dupka près du v.Stradalovo, distr.Kjustendil).

Troglophile? - Proche de l'espèce épigée D.biokovensis Hold. de la Macédoine Yougoslave. Trouvée courant sur l'argile.

Duvalius (Paraduvalius) beroni Guéorguiev, 1971

Vr 42 - Tošova dupka (Guéorguiev, 1971, p.161).

Troglobie.- Un exemplaire a été trouvé courant sur le sable.

Duvalius (Paraduvalius) pretneri Guéorguiev, 1971

Sf 24 - Mečata dupka (Guéorguiev, 1971, p.160).

Troglobie.- Très rare dans la grotte.

Trechus crucifer La Brûlerie, 1875

Sl 24 - Manolovata peštera, 7.VII.1967, P.Tranteev leg. (H.Coiffait det.).

Troglophile.

Trechus austriacus Dejean, 1831

Rs 1 - Orlova čuka, 4.IV.1966, St.Andreev leg. (H.Coiffait det.).

Troglophile.

Trechus subnotatus Dejean, 1831

Rs 1 - Orlova čuka, 4.IV.1966, St.Andreev leg. (H.Coiffait det.). Sm 32 - Djavolskoto gârlo, 30.VI.1966, Hr.Delčev leg. (H.Coiffait det.).

Troglophile.

Trechus obtusus Erichson, 1837

Ja 1 - Bozkite, le 7.VII.1959, P.Beron leg. (H.Coiffait det.).

Trogloxène.

Trechus quadristriatus Schrank, 1781 (H.Coiffait det.).

Pn 4 - Sedlarkata, 10.IX.1968, P.Beron leg. Vr 38 - Bulina dupka, 2.X.1968, id. Vr 69 Pešova jama, 29.V.1967, P.Tranteev leg. Lv 4 - Svirčovica, 16.VIII.1966, Hr.Delčev leg. Lv 5 Bankovica, 18.VIII.1966, id. Lv 49 - Dâlbokata propast, 17.VI.1962, id. Lv 58 - Skravenika, 18.VIII.1966, id. Lv 69 - Enčova dupka, 18.VIII.1966, id.

Trogloxène muscicole.

Trechus cardioderus balcanicus Jeannel, 1927 (H.Coiffait det.) 
B1 2 - Bojčovata peštera, 28.II.1969, P.Beron leg. Ja 1 - Bozkite, 7.VII.1959, P.Beron et A.Popov leg. Lv 4 - Bankovica, 1.V.1963, P.Tranteev leg.; 18.VIII.1966, Hr.Delčev leg.; 21.II.1968, M.Kvartirrikov leg. Rs 1 - Orlova čuka, 4.IV.1966, St.Andreev leg. Sl 12 Lednika, 14.VII.1964, A.Popov leg. Vd 37 - Magura 2, 19.X.1969, P.Beron leg.

Troglophile.

\section{Rambousekiella ledenikensis Knirsch, 1925}

Mg 18 - Grebenjo, 16.VI.1970, 1 \& morte, P.Beron leg. (V.Guéorguiev et P.Beron det.).

Troglobie.- Espèce assez rare.

Pristonychus punctatus Dejean, 1828 (H.Coiffait det.).

Ja 13 - Leleška dupka 2, 8.VIII.1970, P.Beron et V.Beškov leg. Ja 7 - Suhata dupka, 5.VIII.1970, id. Mg 16 - Dupkata, 4.II.1967, P.Beron leg. Pv 8 - Jamata, 27.VIII.1970, Hr.Delčev leg. Pv 12 - Cânkaliva dupka, 27.IV.1969, P.Beron et V.Beškov leg. Pv $13-$ Fidjafkinata dupka, 28.IV.1969, idem. Sn 9 - Krumovi porti, 22.IX.1968, P. Beron leg Vd 29 - Jame 3, 4.X.1967, H. Delčev leg.

Trogloxène régulier (?).

Pristonychus cimmerius Fisch.-Waldh., 1823

Rz 4 - Kaca peštera, 23.VIII.1961, P.Beron et V.Beškov leg. (H.Coiffait det.).

Troglophile guanobie.

Pseudophonus rufipes (Dejean, 1774)

Vr 58 - Moravata (Popov, 1969, p.37).

Trogloxène.

Tachys sp.

Pv 14 - Ivanova voda (Hazelton, 1970, p.36).

Fam. S t a ph y 1 in id a e

Quedius (Microsaurus) mesomelinus skoraszewskyi Korge (H.Coiffait det.)

Bs 4 - Jamata, 9.VIII.1970, P.Beron et V.Beškov leg. Ja 13 - Leleška dupka 2, 8.VIII.1970, id. Kl 4 - Jamkata, 29.X.1966, id. Lv 4 -- Svirčovica, 16.VIII.1966, Hr.Delčev leg. Lv 28 - Toplja, 18.X.1970, P.Beron leg. Lv 31 - Popskata peštera, 21.IV.1965, D. Trifonov leg. Lv 38 - Ptičata dupka, 1.VII.1962, 1.VII.1970, Hr.Delčev leg. Lv 44 - Parnicite, 16.II.1963, P.Beron et V.Beškov leg. Lv 48 - Propast 30, 3.IX.1965, B.Godžunov leg. Lv 53 - Ljubovnata, 20.VIII.1966, Hr.Delčev leg. Lv 62 - Dolnata Čcrvenska peštera, 24.XI.1968, P.Beron leg. Lv 75 - Propast 136, 24.X.1969, id. Mg 8 - Peč, 14.II.1961, V.Guéorguiev leg. Pk 2 - Živata voda, 22.VI.1969, P.Beron leg. Pz 3 - Pešterata, 17.VII.1961, id. Pn 2 - Hajduškata peštera, 9.II.1964, id. Pn 4 - Sedlarkata, 10.IX.1968, id. Pv 4 - Topčika 23.VIII.1970, Hr.Delčev leg. Rs 1 - Orlova čuka, 4.IV.1966. St.Andreev leg. Sl 22 - Žilovata češma, 21.IV.1967, P.Beron 
leg. Sl 25 - Kozjata peštera, 19.X.1966, Hr.Delčev leg. Sm 21 -- Handâka, 4.VIII.1969, id. Sf 8 - Temnata dupka, 22.IX.1962, A.Petkova leg. Sf 12 - Kolibata, \&.X.1958, A.Popov leg. Sf 16 - Otečestvo, 24.V.1959, V.Beškov leg. Sf 29 - Zidanka, 25.VIII.1963, P.Beron et V.Decu leg. Sf 30 - Temnata dupka, 17.III.1957, P.Tranteev leg. Sf 74 - Padež, 1.VI.1969, P.Beron leg. Sn 7 - Peštera 1, 22.IX.1968, id. Vd 2 - Magura, 20.VIII.1963, L. Botoşâneanu et V.Decu leg. Vd 4 - Neprivetlivata, 3.VI.1961, P.Beron leg. Vd 8 - Suhi peč, 25.XII.1969, id. Vd 10 - Tâmni peč, 30.X.1967, Hr.Delčev leg. Vd 14 - Levi Suhi peč, 29.X.1969, P.Beron leg. Vd 16 - Tâmna dupka, 5.X.1967, id. Vd 29 - Jame 3, 4.X.1967, Hr.Delčev leg. Vd 33 - Koritska glama, 22.X.1969, P.Beron leg. Vd 34 - Redaka 2, 22.X.1969, id. Vr 5 - Serapionovata peštera, 2.V.1959, A.Popov leg. Vr 16 - Reznjovete, 23.VIII.1963, V.Decu leg. Vr 17 - Ledenika, 10.IV.1967, P.Tranteev leg. Vr 22 - Ponora, 18.VIII.1963, P.Beron leg. Vr 23 - Golemata jama, 8.VI.1951, Iv.Buresch leg. Vr 31 - Garvanec, 19.VIII.1963, P.Beron leg. Vr 40 - Kalnata dupka, 27.IX.1968, Hr.Delčev leg. Vr 42 - Tošova dupka, 12.XII.1965, V.Beškov leg.; le 17.II.1968, P.Beron leg. Vr 60 - Gâgla, 30.IV.1968, P.Beron leg. Vr 62 - Popudžijnicata, 3.X.1969, id.

Troglophile.- Mr H.Coffait a été aimable de nous faire savoir que "Les autres Quedius mesomelinus déterminés [voir Guéorguiev et Beron, 1962] appartiennent aussi à cette sous-espèce récemment décrite" (In lit.).

Quedius mesomelinus (Marsham, 1802)

Pk 1 - Duhlata (Hazelton, 1970, p.35).

Troglophile.-- Les exemplaires en question appartiennent vraisemblablement aussi à la sous-espèce précédente.

Quedius (M.) fulgidus (Fabricius, 1787)

Sl 15 - Zmejovi dupki (Rambousek, 1909, p.12).

Trogloxène.

Quedius (Sauridus) troglophilus Coiffait, 1969

Lv 5 - Bankovica (Coiffait, 1969, p.46). Lv 67 - Šopa (id.). Lv 68 - Hubavica (id.). Lv 69 Enčova dupka (id.). Sf 62 - Zahlupena dupka (id.).

Troglophile.

Quedius (Sauridus) gueorguievi Coiffait, 1967

Sf 57 - Porojnata dupka (Coiffait, 1967, p.399).

Trogloxène(?).

Quedius (Sauridus) obliteratus Erichson, 1840

Sl 15 - Zmejovi dupki (Rambousek, 1909, p.12).

Trogloxène.

Ocypus globulifer Fourcroix, 1785 
S1 15 - Zmejovi dupki (Rambousek, 1909, p.11, sub. "Staphylinus globulifer Geoffroy"). Trogloxène.

Medon fusculus Mannh., 1849

Sl 15 - Zmejovi dupki (Rambousek, 1909, p.11).

Trogloxène.

Aleochara (Polychara) diversa J.Sahlberg, 1876

Ja 10 - Popovata peštera, 5.VIII.1970, P.Beron et V.Beškov leg. Sl 18 - Subatta, 13.VII.1964, A.Popov leg.

Trogloxène-guanophile.

Philonthus cephalotes Gravenhorst, 1802

Ja 1 - Bozkite, 7.VII.1959, P.Beron et A.Popov leg. Mg 8 - Peč, 14.II.1961, V.Guéorguiev leg. Sf 12 - Kolibata, 5.X.1958, A.Popov leg.

Troglophile-guanophile.

Atheta (Aloconota) sulcifrons Stephens, 1832

Lv 10 - Zadânenka, 14.V.1962, P.Tranteev leg. Lv 5 - Bankovica, 18.VIII.1966, Hr.Delčev leg. Vr 30 - Mladenovata peštera, 17.VIII.1963, P.Beron et V. Decu leg. Vr 38 - Bulina dupka, 2.X.1968, P.Beron leg. Vr 60 - Gâgla, 30.IV.1968, id.

Trogloxène.

Atheta (Atheta) trinotata (Kraatz, 1874)

Pv 14 - Ivanova voda (Hazelton, 1970, p.36).

Trogloxène.

Atheta (Atheta) macroptera Bernhauer, 1913

Ja 4 - Drânči dupka, 6.VIII.1970, P.Beron et V.Beškov leg. K1 1 - Uske, 20.X.1963, id. Kl 4 Jamkata, 29.X.1966, id. Kr 3 - Hasarskata peštera, 16.XII.1962, P.Beron leg. Lv 2 - Hajduška dupka, 3.III.1936, N.Atanasov leg. Lv 5 - Bankovica, 20.VII.1948, Iv.Ivanov leg.; 24.IV.1961, P.Tranteev leg. Lv 9 - Ovnarkata, 19.VIII.1966, Hr.Delčev leg. Lv 20 - Rušovata peštera, 6.V.1964, V.Guéorguiev leg. Lv 34 - Vodopada, 11.VII.1965, P.Tranteev leg. Lv 44 Parnicite, 7.I.1963, A.Petkova leg.; 16.II.1963, V.Beškov leg. Lv 47 - Bezimenna 22, 4.IX.1964 et 28.VII.1967, P.Tranteev leg. Lv 58 - Skravenika, 18.VIII.1966, Hr.Delčev leg. Lv 72 - Peštera 15, 10.VIII.1968. Mg 21 - Srednjata, 11.X.1969, P.Beron leg. Pk 11 - Goljamata peštera, 5.X.1969, V.Eieškov leg. Pv 7 - Gargina dupka, 27.VIII.1970, Hr.Delčev leg. Pv 10 Hralupa, 9.VII.1962, P.Beron leg. Pv 11 - Ledenicata, 6.VII.1962, id. Pv 12 - Cânkaliva dupka, 27.IV.1969, V.Bešk ov et P.Beron leg. Pv 18 - Ahmetjova dupka, 28.VIII.1970, Hr.Delčev leg. Rz 3 - Kulina dupka, 25.VIII.1961, V.Beškov leg. Sf 8 - Temnata dupka, 21.II, 4.IV et 9.V.1965, 6.III.1966, A.Popov leg. Sf 12 - Kolibata, 5.X.1958, id. Sf 30 - Temnata dupka, 17.III.1957, P.'Tranteev leg. Sf 32 - Ražiška peštera, 8.VII.1948, P.Drenski leg. Sf 69 Hajduškata dupka, 25 IX.1966, D.Kirilov leg. Sf 74 - Padež, 1.VI.1969, P.Beron leg. Sl 1 - 
Dolnata maaza, 5.VI.1935, N.Atanasov leg. Sl 12 - Lednika, 30.VIII.1961, P.Beron leg. Sl 18 - Subatta, 13.VII.1961, A.Popov leg. Sm 16 - Boevskata peštera, 6.VIII.1969, Hr.Delčev leg. Sm 26 - Rizovica, 3.VIII.1969, id. Sn 4 - Dipsiskoju, 13.II.1966, K.Kosev leg. Sn 8 Lozevskata peštera, 9.II.1966, K.Kosev leg.; 22.X.1969, P.Beron leg. Tn 10 - Draganovskata peštera, 8.XI.1962, P.Tranteev leg. Vd 10 - Tâmni peč, 2.X.1967. Vr 1 - Prilepnata peštera, 3.V.1961, P.Tranteev leg. Vr 5 - Serapionovata peštera, 2.V.1959, A.Popov leg. Vr 28 Tigančeto, 16.VIII.1963, 14.X.1969, P.Beron leg. Vr 34 - Dupna mogila, 7.II. 1964, P.Beron et A.Popov leg. Vr 42 - Tošova dupka, 17.II.1968, P.Beron leg.

Troglophile - guanobie.

\section{Fam. D y t is c id a e}

Agabus (Dichonectes) guttatus Paykull, 1798

Vd 3 - Cankinoto vrelo, 21.X.1971, P.Beron leg. (V.Guéorguiev det.).

Trogloxène.- Plusieurs dizaines de ces Coléoptères se trouvaient dans les restes du ruisseau qui coule parfois dans la grotte et sort par son entrée.

Ilybius fuliginosus (Fabricius, 1792)

Lv 5 - Bankovica (Hazelton, 1970, p.33).

Trogloxène.

Fam. His te rid a e

Gnathoncus rotundatus Kugel, 1792

Mg 11 - Mitrevska dupka, 26.XII.1969, P.Beron leg. (H.Coiffait det.).

Troglophile? - Il est à noter que G.rotundatus fut trouvé aussi dans le guano des Chiroptères qui habitent la grotte Bachardenskaja peštera en Asie Centrale (U.R.S.S.), où, d'après Kouzjakin, (1950, Letučie miši, Moskva) cette espèce vit aux dépens des coprophages.

\section{Gnathoncus nannetensis Marsham, 1862}

Vr 1 - Prilepnata peštera, 3.V.1961, P.Tranteev leg. (H.Coiffait det.). Troglophile?

Fam. P s e l a ph i d a e

Bryaxis (Erichobythus) beroni Z.Karaman, 1969

Vr 31 - Garvanec (Karaman, 1969, p.60). Vr 42 - Tošova dupka, 17.II.1968, P.Beron leg. (H.Coiffait det.).

Trogloxène.- Quand même, tous les 3 exemplaires connus ont été trouvés dans les grottes. 
Langelandia sp.

Fam. C o l y di i d a e

Pv 13 - Fidjafkinata dupka, 28.IV.1969, P.Beron et V.Beškov leg. (H.Coiffait det.).

Endogé.

Fam. En d o m y c h i d a e

Mycetaea hirta Marsham, 1802

Bs 4 - Jamata, 7.V.1963, P.Beron leg. (H.Coiffait det.). Rs 1 - Orlova čuka, 4.IV.1966, St.Andreev leg. (H.Coiffait det.). Vr 42 - Tošova dupka, 17.II.1968, P.Beron leg. (H.Coiffait det.).

Troglophile.- Vit sur les moisissures.

\section{Fam. Cryptoph agid a e}

Micrambe translatus (Grouvelle, 1916)

Sf 30 - Temnata dupka, 14.III.1960, V.Guéorguiev leg. (H.Coiffait det.). Sf 32 - Râziška peštera, 9.VII.1948, P.Drenski leg.; le 25.XI.1966, P.Beron leg. (H.Coiffait det.).

Troglophile (? ).- On l'a trouvé à $200 \mathrm{~m}$ à l'intérieur de la grotte Râziška peštera sur des excréments.

Cryptophagus cellarius (Scopoli, 1763)

Vd 32 - Djado Stanov Stol, le 12.X.1969, P.Beron leg. (H.Coiffait det.).

Trogloxène.

Cryptophagus scutellatus Newman, 1834

Sf 30 - Temnata dupka, le 14.III.1960, V.Guéorguiev leg. (H.Coiffait det.). Sf 32 - Râziška peštera, 9.VII.1948, P.Drenski leg. (H.Coiffait det.).

Lucifuge.

Ephistemus globulus (Paykull, 1798)

Lv 4 - Svirčovica, 16.V III.1966, Hr.Delčev leg. (H.Coiffait det.).

Trogloxène detriticole.

Fam. C a t o p i d a e (H.Coiffait det.)

Choleva (Cholevopsi:) paskoviensis Reitter, 1913

Vd 19 - Redaka 1 - Gornata, 19.X.1969, P.Beron leg. Vr 63 - Govedarnika, 9.X.1968, id.

Subtroglophile.- Dans les deux grottes les Choleva avaient été assez nombreuses à l'époque de notre visite. 
Choleva agilis Illiger, 1789

Lv 31 - Popskata peštera, 6.X.1925, N.Radev leg. Vr 38 - Bulina dupka, 2.X.1968, P.Beron leg. Vr 36 - Govedarnika, 9.X,1968, id.

Subtroglophile.

Choleva angustata (Fabricius, 1781)

Kl 6 - Meča dupka, 27.II.1969, P.Beron leg. Lv 73 - Graždenica, 25.XI.1968, id.

Subtroglophile.

Choleva oblonga Latreille, 1807

Ja 1 - Bozkite, 7.VII.1959, P.Beron et A.Popov leg. Vr 63 - Govedarnika, 9.XI.1968, P.Beron leg.

Subtroglophile.- Espèce souvent pholéophile.

Choleva sp.

Lv 5 - Bankovica (Hazelton, 1970, p.33).

Nargus badius (Sturm, 1839)

Mg 2 - Levica vreloto, 17.IX.1964, P.Beron et V.Beškov leg.

Subtroglophile.- Motaş et al. (1967, Ann. de Spéléol., XXII) ont trouvé cette espèce dans deux grottes de Roumanie "en très grand nombre près des ouvertures, sous les plaques calcaires mêlées de détritus végétal et de terre". Nos exemplaires ont été récoltés dans des conditions identiques.

Catops coracinus (Kelln., 1846)

Vr 23 - Golemata jama, 8.VI.1951, Iv. Buresch leg.

Trogloxène (? ).

Catops picipes (Fabricius, 1792)

Vr 17 - Ledenika, 10.VIII.1962, V.Guéorguiev leg.

$$
\text { D i p ter a }
$$

Fam. M y c e t o philidae

Speolepta leptogaster Winnertz, 1863

Pv 14 - Ivanova voda (Hazelton, 1970, p.36).

Troglophile. 
Fam. L i m n ob i id a e

\section{Limnobia ǹubeculosa L.}

Pv 14 - Ivanova vodia (Hazelton, 1970, p.37).

Troglophile.

Fam. Trich oc e rida e

\section{Trichocera regelationis L.}

Pv 14 - Ivanova vodal (Hazelton, 1970, p.35).

Trogloxène.

Fam. S t r a t i o m y i d a

Actina nitens (Latreille, 1809)

Vr 17 - Ledenika (Beshovski, 1972, p.22)

Trogloxène.

Fam. D o l i c h o p o d id a e

Liancalus virens Scopoli, 1763

Pn 2 - Hajduškata peštera (Beshovski, 1972, p.22)

Trogloxène.

\section{Fam. H e 1 o m y s id a e}

Thelida atricornis Meigen, 1830

Lv 17 - Dolnata peštera (id.). Lv 18 - Sâeva dupka (id.). Lv 24 - Golemata peštera (id.). Pn 1 - Kajalâškata peštera (id.). Gb 2 - Bačo Kiro (id.). Sf 12 - Kolibata (id.). Sf 22 - Golaškata peštera (id.).

Troglophile.

Thelida rotundicornis Zetterstedt, 1846

Gb 2 - Bačo Kiro (Beshovski, 1972, p.23)

Trogloxène.

Eccoptomera emarginata Loew, 1862

Gb 1 - Andâka (Beshovski, 1972, p.23) Sm 3 - Dupkata (id.). Vr 17 - Ledenika (id.).

Subtroglophile. 
Eccoptomera obscura Meigen, 1830

Pv 14 - Ivanova voda (Hazelton, 1970, p.36).

Trogloxène.

Acantholeria cineraria (Loew, 1862)

Sf 5 - Krivata pešt (Beshovski, 1972, p.23)

Trogloxène.

Scoliocentra (Amaebaleria) brachypterna Loew, 1873

Sf 30 - Temnata dupka (Beshovski, 1972, p.23)

Trogloxène.

Scoliocentra (Amaebaleria) caesia Meigen, 1830

Gb 2 - Bačo Kiro (Beshovski, 1972, p.23) Sm 3 - Dupkata (id.). Sl 1 - Dolnata maaza (id.). Trogloxène régulier.

Scoliocentra (Amaebaleria) ventricosa Becker, 1907

Gb 1 - Andâka (Beshovski, 1972, p.23) Gb 2 - Bačo Kiro (id.).

Trogloxène.

Helomyza captiosa Gorodkov, 1962

Gb 2 - Bačo Kiro (Beshovski, 1972, p.23). Lv 18 - Sâeva dupka (id.). Pz 4 - Novata peštera (id.). Sf 25 - Javoreckata peštera (id.). Sf 52 - Vodnata peštera (id.). Sf 4 - Svetata voda (id.). Sf 5 - Krivata pešt (id.). Sl $15-$ Zmejovi dupki (id.). Sm 4 - Sbirkovata peštera (id.). Sm $6-$ Čelečkata peštera (id.). Tn 3 - Golema Podlisca (id.).

Troglophile.- D'après Beshovski (1972), un bon nombre des Diptères bulgares, déterminées comme Helomyza serrata, vont s'avérer appartenant à l'espèce récemment décrite H.captiosa Gor.

Helomyza serrata L.

Tn 6 - Trošana (Beshovski, 1972, p.24).

Trogloxène.

Fam. Chi r o m y i d a e

Chiromyia flava (L.)

Sf 12 - Kolibata (Beshovski, 1972, p.24).

Trogloxène. 
Fam. S p h a r o c e rida e

Limosina racovitzai Bezzi, 1911

Pv 14 - Ivanova vodla (Hazelton, 1970, p.36, avec "? "). Lv 2 - Hajduška dupka (Beshovski, 1972, p.24). Lv 20 -- Rušovata peštera (id.). Gb 7 - Izvora (id.). Sf 32 - Ražiška peštera (id.). Vr 1 - Prilepnata pestera (id.).

Trogloxène.

Limosina silvatica (Meigen, 1830)

Lv 18 - Sâeva dupka (Bešovski, 1972).

Trogloxène.

Fam. D r os o p hilida e

Phortica variegata (Fallén, 1823)

Vr 3 - Studenata dupka (Bešovski, (1972).

Trogloxène.

Drosophila fasciata Meigen, 1830

Lv 2 - Hajduška dupka (Bešovski, 1972). Pz 4 - Novata peštera (id.). Sf 30 - Temnata dupka (id.). Sf 41 - Čavkite (id.).

Trogloxène

Fam. Ph or id a e

Triphleba antricola Schmitz, 1918

Pk 1 - Duhlata (Hazelton, 1970, p.34).

Troglophile-guanophile

Megaselia sp.

Lv 4 - Svirčovica (Hazelton, 1970, p.33).

Fam. Ny c te ribiidae

Penicillidia dufouri Westwood, 1835

Pv 14 - Ivanova voda (Hazelton, 1970, p.37).

Parasite des Chiroptères. 
Lasius niger L.

Vd 46 - Falkovskata peštera, 18.X.1971, P.Beron leg. (N.Atanasov det.).

Trogloxène.

Lasius brunneus Latreille, 1798

Vd 50 - Zankovska peštera, 27.X.1971, P.Beron leg. (N.Atanasov det.).

Trogloxène.

Siph on a p ter a

Fam. Is ch nopsy 11 id a e

Nycteridopsylla ancyluris johanae Hurka, 1970

Sf 32 - Ražiška dupka (Hurka, 1970, p.21).

Parasite.- Trouvée sur Pipistrellus pipistrellus.

$$
\begin{gathered}
\text { Lepidoptera } \\
\text { Fam. Acrolepiidae }
\end{gathered}
$$

Acrolepia granitella Treitschke, 1833

Sf 30 - Temnata dupka (Skalski, 1971, p.215).

Trogloxène régulier.

Acrolepia pulicariae Klimesch, 1956

Sf 30 - Temnata dupka (Skalski, 1971, p.216). Sf 34 - Kozarskata peštera (id.).

Trogloxène.

Fam. N oc tu id a e

Antophila limbata Staudinger, 1870

Sf 30 - Temnata dupka (Skalski, 1971, p.216). Sf 32 - Ražiška dupka (id.).

Trogloxène.- Comme pour l'espèce précédente, ces localités sont les premières en Bulgarie.

\section{Scoliopteryx libatrix L.}

Sf 30 - Temnata dupka (Slivov, 1968, p.172). Bs 5 - Ezeroto, le 7.V.1963, P.Beron (observ.). 
Mg 20 - Vodnata dupka, 24.XII.1969, id. Vd 38 - Stanišina dupka, 26.X.1971, id. Vd 48 Dupkite, 29.X.1971, id.

Subtroglophile.

Fam. G e o m e trid a e

\section{Triphosa dubitata $\mathrm{L}$.}

Sf 30 - Temnata dupka (Slivov, 1968, p.177).

Subtroglophile.

Truphosa sabaudiata Dupouchel, 1827

Mg 22 - Veneco 1 - Gornjata, 11.X.1969, P.Beron (observ.). Vd 38 - Stanišina dupka, 26.X.1971, id. Vd 42 - Golema dupka, 23.X.1971, id. Vd 43 - Dupkata v Prosečeno, 26.X.1971, id.

Subtroglophile.- Très commune dans les grottes bulgares, paraît plus nombreuse que T.dubitata.

\section{Trichoptera}

Fam. Hy d ropsychidae

Hydropsyche instabilis (Curtis, 1834)

Sm 32 - Djavolsk oto gârlo (Kumanski, 1971, p.209).

Trogloxène.- Les deux exemplaires trouvés étaient sur les parois humides à $200 \mathrm{~m}$. à l'intérieur de la grotte.

Fam. Li m ne philida e

Stenophylax vibex speluncarum Mc Lachlan, 1875

Sf 3 - Dinevata pešt (Kumanski, 1971, p.210). Sf 4 - Svetata voda (id.). Sf 38 - Kitova kukla (id.). Sm 18 - Sančova dupka (id.). Šn 6 - Zandana (id.). Sl 23 - Čerešata (id.).

Trogloxène régulier.- D’après Kumanski (1971), tous les Stenophylax vibex des grottes bulgares appartiennent à la sous-espèce S.vibex speluncarum et rion pas à la sous-espèce typique.

Stenophylax mitis Mc Lachlan, 1875

Sf 38 - Kitova kukla (Kumanski, 1971a, p.210). Sl 6 - Kipilovskata peštera (id.). Vd 20 Mečata dupka (id.). Vd 29 - Jame 3 (id.).

Trogloxène régulier.

Micropterna nycterobia Mc Lachlan, 1875

Lv 5 - Bankovica (Kumanski, 1971a, p.210). Lv 58 - Skravenika (id.). Pk 10 - Dimova jamka (id.). Sf 3 - Dinevata pešt (id.). Sf 4 - Svetata voda (id.). Sf 5 - Krivata pešt (id.). Sf 26 - 
Svârdelo (id.). Sf 47 - Vodnata pešt (id.). Sm 18 - Sančova dupka (id.). Sm 22 - Mečata dupka (id.). Šn 7 - Peštera 1 (id.). Vd 21 - Prelaz (id.). Vd 29 - Jame 3 (id.).

Trogloxène régulier.

Micropterna sequax Mc Lachlan, 1875

Sf 3 - Dinevata pešt (Kumanski, 1971a, p.210). Sl 24 - Manolovata peštera (id.). Sm 1 Lednicata (id.). Sm 18 - Sančova dupka (id.).

Trogloxène régulier.

Micropterna malaspina Schmid, 1957

Sn 4 - Dipsiskoju (Kumanski, 1971, p.105). Sz 3 - Toplata dupka (Kumanski, 1971a, p.211). Grotte près de Sumen (= Sn 4 ? )(id.).

Trogloxène regulier.- Cette espèce assez intéressante au point de vue zoogéographique a été décrite d'Asie Mineure.

Micropterna fissa Mc Lachlan, 1875

Sm 29 - Golobovica 3 (Kumanski, 1971a, p.211).

Trogloxène régulier.- Semble être rare dans les grottes bulgares mais par contre prédomine en Italie.

Mesophylax aspersus (Rambour, 1842)

Sv 58 - Skravenika (Kumanski, 1971a, p.211). Sf 47 - Vodnata pešt (id.).

Trogloxène régulier.

Fam. Ph il o p ot a mid a e

Philopotamus montanus Donovan, 1813

Pz 1 - Lepenica (Radovanovičc, 1953).

Trogloxène occasionnel.- Cette espèce a été omise dans les deux listes précédentes (1962 et 1967). Elle est mentionnée par Radovanovic sub "grotte près de Lâdžane".

\section{Vertebrata \\ Aves}

Fam. C o r vid a e

Pyrrhocorax graculus L.

Sf 74 - Padež, 1.VI.1969, P.Beron (observ.). 


\section{Hirundo rustica L.}

Fam. H i r u n d in i d a e

Ja 6 - Karaburnu, 6.VIII.1970, P.Beron et V.Bešk ov (observ.).

\section{Mammalia}

\section{Ch iroptera}

Fam. Rh in olophid a e

\section{Rhinolophus ferrumequinum (Schreber, 1774)}

Bs 7 - Kirečnicata (Beron, 1968, p.160). Lv 52 - Sinjoto kolelo (Beron, 1970, p.147). Vr 48 Ezeroto (id. p.145). Sm 31 - Ulcata (Beron, 1968, p.158). K1 4 - Jamkata (id.). Vr 19 Golemata Meča dupka (id.). Šn 9 - Krumovi porti (Beron, 1970, p.146). Sm 28 - Golobovica 2, 3.IV.1967, P.Beron (observ.). Sm 13 - Imamova dupka (Anon., 1972, p.41). Sm 18 Sančova dupka (id.). Bs 5 - Ezeroto, 7.V.1963, P.Beron (observ.). Pk 10 - Dimova jamka, 5.X.1969, V.Beškov (observ.). Vd 14 - Levi Suhi peč, 27.I.1971 (id.). Vd 22 - Kračimirskoto vrelo, 23.X.1971, P.Beron (observ.). Vd 40 - Tatarska dupka, 25.X.1971 (id.). Vd 43 Golema dupka, 23.X.1971 (id.). Vd 46 - Falkovskata peštera , 18.X,1971 (id.). Vd 49 Mejuva dupka 1, 27.X.1971 (id.).

Rhinolophus mehelyi Matchie, 1901

Pn 7 - Nanin kamâk, G.VIII.1971, P. Beron et V.Beškov (observ.).

Rhinolophus euryale Blasius, 1853

Pk 1 - Duhlata (Hazclton, 1970, p.35).

\section{Rhinolophus blasii F'eters, 1866}

Pk 9 - Jamkata (Beron, 1968, p.158). Vr 8 - Samuilica (id., p.160). Mg 12 - Grimnena dupka, 29.X.1971, P.Beron (observ.).

Rhinolophus hipposideros (Bechstein, 1800)

Bs 5 - Ezeroto, 2.IV.1971, V.Beškov (observ.). Pk 4 - Filipovskata peštera (Beron, 1968, p.158). Mg 16 - Dupkata (Beron, 1970, p.145). Lv 77 - Borova dupka 2, 1970, P.Beron (observ.). Mg 12 - Grimnena dupka, 29.X.1971 (id.). Vd 39 - Zemna dupka, 26.X.1971 (id.). Vd 40 - Tatarska dupka, 25.X,1971 (id.). Vd 41 - Medžak dupka (id.). Vd 44 - Zmijskata propast, 28.X.1971, P.Eeron (observ.).

Fam. Ves pertilion id a e

Myotis daubentoni (Kühl, 1819)

Pn 7 - Nanin kamâk, 28.I.1971, V.Beskov (observ.). 
Myotis emarginatus (Geoffroy, 1806)

Sm 1 - Ledenicata (Anon., 1971, p.41). Sm 13 - Imamova dupka (id.). Sm 20 - Gorna Karanska dupka (id.). Sm 18 - Sančova dupka (id.).

Myotis nattereri (Kühl, 1818)

Sm 20 - Gorna Karanska dupka (Anon., 1971, p.41).

Myotis capaccinii (Bonaparte, 1837)

Pn 7 - Nanin kamâk, 9.VIII.1971, P.Beron (observ.). Vd 8 - Suhi peč, 27.XII.1969 (id.).

Myotis mystacinus (Kühl, 1819)

Sm 1 - Ledenicata (Anon., 1971, p.41). Sm 4 - Sbirkovata peštera (id.). Sm 5 - Samurski dupki (id.).

Myotis bechsteini (Kühl, 1818)

Sm 13 - Imamova dupka (Anon., 1971, p.42).

Myotis blythi oxygnathus (Monticelli, 1885)

Vr 19 - Golemata Meča dupka (Beron, 1968, p.159). Vr 52 - Sokolskata dupka (id. p.158). Pv 14 - Ivanova voda (Hazelton, 1970, p.35). Sm 1 - Ledenicata (Anon., 1971, p.42). Pv 7 Gargina dupka (Beron, 1971, p.144). Pn 7 - Nanin kamâk, 9.VIII.1971, P.Beron (observ.). Vd 18 - Studena, 19.X.1969 (id.).

Myotis myotis (Borkhausen, 1797)

Vr 52 - Sokolskata dupka (Beron, 1968, p.158). Sm 26 - Červena dupka (id.). Pn 5 - Genina peštera (Beron, 1970, p.146). Pv 14 - Ivanova voda (Hazelton, 1970, p.35). Sm 1 - Ledenicata (Anon., 1971, p.42). Sm 5 - Samurski dupki (id.). Sm 18 - Sančova dupka (id.). Sm $19-$ Dolna Karanska dupka (id.). Mg 12 - Grimnena dupka, 29.X.1971, P.Beron (observ.). Pn 7 Nanin kamâk, 9.VIII.1971 (id.). Vd 7 - Propast, 20.X.1971 (id.). Vd 18 - Studena, 19.X.1969 (id.). Vd 46 - Falkovskata peštera, 18.X.1971 (id.).

Myotis brandti (Eversmann, 1845)

Sm 1 - Ledenicata (Anon., 1971, p.42).

Miniopterus schreibersi (Kühl, 1819)

Pn 5 - Genina peštera (Beron, 1970, p.145). Mg 12 - Grimnena dupka, 29.X.1971, P.Beron (observ.). Pn 7 - Nanin kamâk, 9.VIII.1971 (id.). Vd 1 - Vârkan, 20.X.1969 (id.). Vd 7 Propast, 20.X.1969 (id.). Vd 18 - Studena, 19.X.1969 (id.). Vd 22 - Kračimirskoto vrelo, 23.X.1971 (id.). Vd 42 - Golema dupka, 23.X.1971 (id.). 
Barbastella barbasteilus (Schreber, 1774)

Sm 1 - Ledenicata (Aron., 1971, p.42).

Plecotus austriacus (Fischer, 1829)

Vr 57 - Starata Prodânka (Beron, 1970, p.145).

Plecotus auritus L.

Sm 1 - Ledenicata (Anon., 1971, p.42). Sm 5 - Samurski dupki (id.).

Eptesicus serotinus (Schreber, 1775)

Sm 1 - Ledenicata (A.non., 1971, p.42). Sm 13 - Imamova dupka (id.). Sm 18 - Sančova dupka (id.). Sm 32 - Ražiška dupka, 2.XI.1971, P.Beron (observ.).

Carnivora

Fam. F e 1 id a e

Felis silvestris Schreber, 1777

Vd 8 - Suhi peč, 27.XII.1969, P.Beron et V.Beškov (observ.).

Le chat sauvage a été vu à $100 \mathrm{~m}$ à l'in térieur de la grotte.

\section{DESCRIPTIONS NOUVELLES ET SUPPLEMENTAIRES DES CAVITÉS BULGARES EXPLORÉES AVEC ÉNUMÉRATION DE LEUR FAUNE}

\section{Cavités dans le district de Blagoevgrad - Bl}

Bl 1. Manailovata peštera - grotte près du v.Osikovo: Stygiosoma beroni

*Bl 4. Bojčova peštera - grotte près du v.Stanke Lisičkovo. Humide, large entrée, quelques chambres presque obscures: Trechus cardioderus balcanicus

*Bl 5. Bânderica - grotte-gouffre dans la montagne Pirin, près du châlet Bânderica. longueur totale $430 \mathrm{~m}$, dénivellation $-120 \mathrm{~m}$. Un ruisseau y entre et coule vers le fond: Lepthyphantes rhodopensis

\section{Cavités dans le district de Burgas - Bs}

Bs 3. Kačulskata propast - gouffre près du v.Gramatikovo: Rhinolophus ferrumequinum

Bs 4. Jamata - grotte près du v.Mladežko, au lieu-dit "Kaleto". Après une descente 
de $7 \mathrm{~m}$, on entre dans la galerie principale, longue de $140 \mathrm{~m}$. Longueur totale 275 m. Argile, flaques, plusieurs concrétions stalagmitiques: Porrhomma convexum, Nesticus cellulanus

Bs 5. Ezeroto - grotte près du v.Mladežko. Grotte à 2 entrées, humide, un lac au fond. Longueur totale $31 \mathrm{~m}$ : Nesticus cellulanus; Scoliopteryx libatrix; Rhinolophus ferrumequinum, Rh.hipposideros

*Bs 6. Izvornata peštera - grotte près du v.Mladežko. Diaclase, un ruisseau coule au fond. Guano, concrétions. Longueur totale $22 \mathrm{~m}$ : Oxychilus moussoni; Nesticus cellulanus; Monotarsobius bifidus; Micropterna sequax

*Bs 7. Kirečnicata (Sârpijskata dupka) - grotte dans la région du v.Kosti, sur la rive gauche de Rezovska reka, au lieu dit "Sârpiite". Guano, concrétions. Longueur quelques dizaines de m: Vertiginidae; Trichoniscus valkanovi; Rhinolophus ferrumequinum

\section{Cavités dans le district de Gabrovo - Gb}

Gb 1. Andǎka - grotte près de Drjanovski manastir. Longueur totale $1800 \mathrm{~m}$ : Eccoptomera emarginata, Scoliocentra ventricosa

Gb 2. Bačo Kiro - grotte près de Drjanovski manastir. Corr.: longueur totale $2400 \mathrm{~m}$. Thelida atricornis, Scoliocentra caesia, S. ventricosa, Helomyza captiosa

*Gb 7. Izvora - grotte à $3,5 \mathrm{~km}$ à l'Ouest du v.Jantra, sur la rive gauche de la rivière Jantra. Entrée large de $7 \mathrm{~m}$, haute de 3,5 m. Quelques galeries d'une longueur totale de $810 \mathrm{~m}$. Longueur de l'axe principale $476 \mathrm{~m}$ : Meta menardi, M. merianae; Limosina racovitzai

*Gb 8. Ivankinata peštera - grotte près de Gabrovo: Meta menardi

\section{Cavités dans le district de Jambol - Ja}

Ja 1. Bozkite - grotte près du v.Mramor: Porrhomma convexum; Trechus cardioderus balcanicus, T. obtusus, Oxytelus sculpturatus, Choleva oblonga

Ja 2. Stara Sveta Trojca - grotte près du v.Mramor, sur la colline près du monastère "Sveta Trojca". Une seule cavité semi-éclairée avec quelques petits embranchements sombres. Sèche: Meta bourneti, Lepthyphantes leprosus

*Ja 3. Tjasnata propast - gouffre-grotte près du v.Mramor, sur la rive gauche de la rivière Manastirska reka, vis-à-vis du gouffre Drânči dupka. Première partie verticale et étroite, profonde de quelques $6-7 \mathrm{~m}$, suivie de quelques embranchements horizontaux d'une longueur totale d'env. $30 \mathrm{~m}$. Concrétions stalagmitiques, plusieurs racines de plantes: Meta bourneti; Lithobius tjasnatensis

*Ja 4. Drânči dupka - gouffre près du v.Mramor. Deux puits et une galerie principale, dont le sol est recouvert de guano. Dénivellation totale $80 \mathrm{~m}$ : Meta bourneti; Atheta macroptera

*) Les cavités marquées avec une astérisque ne figurent pas dans les deux listes précédentes (1962 et 1967). 
*Ja 5. Drânči dupka (Morfel) - gouffre près du v.Melnica, en dessus du monastère. Dénivellation totale $28 \mathrm{~m}$. Au fond un lac-siphon, profond de plus de $4 \mathrm{~m}$ : Porrhomma convexum, Nesticus cellulanus

*Ja 6. Karaburnu - petit gouffre près du v.Melnica. Profondeur $9 \mathrm{~m}$, le fond sémi-éclairé: Meta bourneti; Hirundo rustica

*Ja 7. Suhata dupka - grotte-gouffre près du v.Melnica, au dessus du monastère. Sèche, longueur 31. m, un ruisseau inaccessible coule au fond: Meta bourneti; Pristonychus punctatus

*Ja 8 . Kesadžijca - grotte-gouffre près du v.Melnica. Humide, beaucoup de guano et d'argile, quelques flaques. Plusieurs embranchements: Lepthyphantes insignis; Lithobius piceus

*Ja 9. Sirokija kajrjak - grotte près du v.Goljam Dervent, à 1,5 km de la frontière avec la Turquie. Argile, plusieurs morceaux de bois pourri. Quelques tortues mortes. Longue env. 25-30 m: Nesticus cellulanus

*Ja 10. Popovata peštera - grotte près du v.Goljam Dervent. Difficile à trouver, dans une forêt de chênes. Humide, galerie simple, longue $16 \mathrm{~m}$ : Aleochara diversa *Ja 11. Vetata straža - gouffre-grotte près du v.Goljam Dervent: Quedius ochripennis, Trechus quadristriatus

*Ja 12. Leleška dupka 1 - grotte près du v.Iglika: Porrhomma convexum

*Ja 13. Leleška dupka 2 - grotte près du v.Iglika: Meta merianae; Quedius mesomelinus skoraszewskyi, Pristonichus punctatus

*Ja 14. Leleška dupka 3 - grotte près du v.Iglika: Oxychilus urbanskii (?)

*Ja 15. Jamata (Goljamata jama) - gouffre-grotte à $4 \mathrm{~km}$ du v.Stefan Karadžovo. Longueur totale $32 \mathrm{~m}$. Accumulations de $\mathrm{CO}_{2}$ au fond: Oxychilus hydatinus (?); Porrhomma convexum; Rhinolophus hipposideros

*Ja 16. Ostâr kamâk - grotte près du v.Mramor, non loin du monastère "Sveta Trojca". Sèche, longue d'env. 25 m: Meta bourneti

\section{Cavités dans le district de Kjustendil - Kl}

Kl 1. Uske - grotte près du v.Cetirci: Atheta macroptera

*Kl 2. Boboševskata peštera - grotte près du v.Boboševo. Longueur env. 25 m: Hoplopholcus forskali, Tegenaria domestica

*KI 3. Ilijskata peštera - grotte près du v.Ilija. Longueur $150 \mathrm{~m}$. Argile: Lepthyphantes spelaeorum. Meta menardi

*Kl 4. Jamkata - grotte près du v.Trekljano. Labyrinthe descendant d'une longueur totale de $200 \mathrm{~m}$ env. et d'une dénivellation de $36 \mathrm{~m}$ : Brachydesmus cristofer; Quedius mesomelinus skoraszewskyi, Atheta macroptera

*Kl 5. Kacite - grotte près du v.Stradalovo. Longue d'env. $20 \mathrm{~m}$ : Tegenaria domestica

*KI 6. Meča dupka - gouffre près du v.Stradalovo. Humide, quelques flaques au fond: Oxychilus glaber striarius; Nesticus cellulanus; Duvalius beshkovi, Choleva angustata

*Kl 7. Vâlči dol - grotte près du v.Gorna Rakovica: Meta menardi 


\section{Cavités dans le district de Kârdžali - Kr}

Kr 1. Tilki ini - grotte près du v.Ostrovica: Meta menardi

Kr 3. Hasarskata peštera - grotte près du v.Gorna Snežinka: Monocyphoniscus bulgaricus; Atheta macroptera

\section{Cavités dans le district de Loveč - Lv}

Lv 2. Hajduškata dupka - grotte près de la gare Karlukovo: Meta merianae, Lepthyphantes leprosus; Cryptops hortensis; Limosina racovitzai, Drosophila fasciata; Atheta macroptera

Lv 4. Svirčovica - grotte près du v.Karlukovo: Diplocephalus cristatus, Tegenaria silvestris, Meta menardi, Nesticus cellulanus; Rhagidia spelaea, Neotrombicula autumnalis; Onychiurus sp., Oncopodura sp. Arrhopalites pygmaeus, Orchesella cf alticola; Megaselia sp.; Quedius mesomelinus skoraszewskyi, Trechus quadristriatus, Ephistemus globulus

Lv 5. Bankovica - gouffre-grotte près du v.Karlukovo: Daudebardia rufa, Atopia quiccardi, Clausilia sp., Monacha haussknechti; Acanthocyclops viridis; Niphargus sp.; Tracheoniscus bulgaricus, Armadillidium vulgare; Nesticus cellulanus, Lepthyphantes spelaeorum, Porrhomma sp.; Heteromurus nitidus, Hypogastrura cf tullbergi, Folsomia sp., Pseudosinella $c f$. fallax, Oncopodura sp., Neelidae gen.sp.; Trechus cardioderus balcanicus, T.quadristriatus, Atheta macroptera, A.sulcifrons, Quedius troglophilus, Illybius fuliginosus, Choleva sp.; Micropterna nycterobia

Lv 7. Temnata dupka - grotte près du v.Karlukovo: Nesticus cellulamus

Lv 9. Ovnarkata - grotte près du v.Karlukovo: Atheta macroptera

Lv 10. Zadânenka - grotte près du v.Karlukovo: Nesticus cellulanus; Atheta sulcifrons

Lv 12. Ledenicata - gouffre près du v.Karlukovo: Lepthyphantes leprosus

Lv 16. Temnata dupka - grotte près de Lukovit: Tracheoniscus bulgaricus

Lv 17. Dolnata peštera - grotte près du v.Zlatna Panega: Hoplopholcus forskali, Lepthyphantes leprosus; Thelida atricornis

Lv 18. Sâeva dupka - grotte près du v.Brestnica: Thelida atricornis, Helomyza captiosa, Limosina silvatica

Lv 20. Rušovata peštera - grotte près du v.Gradešnica: Oxychilus depressus; Trachysphaera costata; Lithobius beshkovi; Atheta macroptera; Limosina racovitzai Lv 24. Golemata peštera - grotte près du v.Mikre: Thelida atricornis

Lv 28. Toplja - grotte près du v.Goljama Železna: Lithobius beshkovi; Quedius mesomelinus skoraczewskyi

Lv 30. Stâlbica - gouffre près du v.Kârpačevo: Niphargus ablaskiri georgievi

Lv 31. Popskata peštera - grotte près du v.Krušuna: Trichoniscus sp.; Typhloiulus georgievi; Quedius mesomelinus skoraszewski, Choleva agilis

Lv 34. Vodopada - grotte près du v.Krušuna. Corr.: longueur explorée $2050 \mathrm{~m}$ : Hoplopholcus forskali, Nesticus cellulanus; Atheta macroptera

Lv 35. Uruška maara - grotte près du v.Krušuna. Corr.: longueur totale $1610 \mathrm{~m}$ : Nesticus cellulanus, Meta menardi 
Lv 36. Futjovskata peštera - grotte près du v.Kârpačevo: Armadillidium klugi

Lv 37. Devetaškata peštera - grotte près du v.Devetaki: Lithobius popovi

Lv 38. Ptičata dupka - gouffre près du v.Černi Osâm: Quedius mesomelinus skoraszewskyi

Lv 44. Parnicite - grotte près du v.Bežanovo: Oxychilus glaber striarius; Hyloniscus riparius, Balkanoniscus minimus, Beroniscus capreolus; Nesticus cellulanus; Polydesmus mediterraneus valachicus; Quedius mesomelinus skoraszewskyi, Atheta macroptera

Lv 47. Bezimenna 22 - grotte près du v.Karlukovo: Tricyphoniscus bureschi, Trichoniscus bureschi; Protoleptoneta bulgarica; Typhloiulus bureschi; Atheta macroptera

Lv 48. Propast 30 (Bezimenna 30) - gouffre près du v.Karlukovo: Polydesmus complanatus illyricus; Lepthyphantes spelaeorum; Quedius mesomelinus skoraszewskyi

Lv 49. Dâlbokata propast - gouffre près du v.Karlukovo: Lepthyphantes spelaeorum, L.centromeroides; Trechus cardioderus balcanicus

*Lv 50. Ćerdženica - grotte près du v.Karlukovo. Humide, longue de $45 \mathrm{~m}$ : Tricyphoniscus bureschi, Trichoniscus bureschi

*Lv 51. Djado Draganovata peštera -- grotte dans la ville Teteven. Argile humide, quelques flaques. Longueur $120 \mathrm{~m}$ : Trichoniscus bureschi; Lithobius agilis

*Lv 52. Sinjoto kolelo - grotte dans la ville Teteven. Humide, à deux étages, longueur totale $300 \mathrm{~m}$; des débris ligneux en quantité: Lepthyphantes balcanica; Rhinolophus ferrurnequinum

*Lv 53. Ljubovnata - gouffre-grotte près du v.Karlukovo. A deux étages, longueur $44 \mathrm{~m}$, dénivellation $-39 \mathrm{~m}$ : Haplophthalmus danicus; Lepthyphantes tenuis, L.spelaeorum, Nesticus cellulanus; Quedius mesomelinus skoraszewskyi

*Lv 54. Zalâmska peštera - grotte près du v.Karlukovo. Longueur $56 \mathrm{~m}$, descendante, boueuse; source périodique dans la grotte: Nesticus cellulanus, Meta menardi * Lv 55. Peštera 4 - gouffre près du v.Karlukovo, au lieu dit Pavlovskoto. Profondeur $-18 \mathrm{~m}$ : Hoplopholcus forskali

* Lv 56. Propast 65 - gouffre-grotte près du v.Karlukovo, au lieu dit Pavlovskoto. Sèche, descendante, longue de $45 \mathrm{~m}$, déniv. $-18 \mathrm{~m}$ : Meta menardi

*Lv 57. Gurleva (Ciurljova) dupka - grotte près du v.Krušuna, au lieu-dit Beltinski dol. Longueur $94 \mathrm{~m}$. Gours, argile: Centromerus jacksoni

*Lv 58. Skravenika - grotte près du v.Karlukovo. Longueur $70 \mathrm{~m}$, un ruisseau y coule: Niphargus bureschi; Nesticus cellulanus, Meta menardi, M.merianae; Trechus quadristriatus, Atheta macroptera; Micropterna nycterobia, Mesophylax aspersus

*Lv 59. Goljamata jama - gouffre près de Teteven, au sommet de la colline Červen. Humide, un puits, profond de $32 \mathrm{~m}$ : Tomocerus minor, Lathriopyga phlegraea, Onychiurus tuberculatus

*Lv 60. Bojkina pieštera - grotte près du v.Karlukovo. Sèche, longue de $42 \mathrm{~m}$, déniv. $-10 \mathrm{~m}$ : Meta menardi

*Lv 61. Propast 71 - gouffre près du v.Karlukovo, nommé aussi "Kaminata". Ce gouffre, dont la profondeur est $-16 \mathrm{~m}$ et la longueur totale $23 \mathrm{~m}$ se trouve au lieu-dit Pavlovskoto: Meta menardi 
*Lv 62. Dolnata Červenska peštera - grotte sur la colline Červen près de Teteven: Meta menardi: Quedius mesomelinus skoraszewskyi

* Lv 63. Gornata Červenska peštera - grotte près de Teteven, quelques mêtres en dessus de Lv 62: Meta menardi

*Lv 64. Malkata jama - gouffre près de Teteven, sur la colline Červen: Oxychilus glaber striarius; Lepthyphantes spelaeorum; Pseudosinella sp.

*Lv 65. Meča dupka - grotte près du v.Gložena. Une galerie de $20 \mathrm{~m}$ de longueur et un gouffre, profond de $30 \mathrm{~m}$ : Meta menardi

*Lv 66. Svinštica - grotte près du v.Gložene. Humide, longue de $35 \mathrm{~m}$ : Oxychilus glaber striarius; Meta menardi

*Lv 67. Šopa - gouffre près du v.Karlukovo. Un puits de $28 \mathrm{~m}$ et un couloir de $15 \mathrm{~m}$, argile humide: Quedius troglophilus

* Lv 68. Hubavica - gouffre près du v.Karlukovo, profondeur $-35 \mathrm{~m}$, argile humide: Quedius troglophilus

*Lv 69. Enčova dupka (Enjuva dupka) - gouffre près du v.Karlukovo. On descend env. $12 \mathrm{~m}$ et l'on arrive dans une galerie, longue de $35 \mathrm{~m}$. Un ruisseau y coule: Nesticus cellulanus; Trechus quadristriatus, Quedius troglophilus

*Lv 70. Sopotskata peštera - grotte près du v.Sopot. Etroite, longueur totale 1186 m: Oxychilus glaber striarius; Typhloiulus bureschi

*Lv 71. Vlaevskata peštera - grotte près du v.Černi Vit, dans la carrière du v.Polaten. Un ruisseau, plusieurs flaques. Humide. Longueur env.200 m: Pseudocypus picipenis bulgaricus

*Lv 72. Tavančeto (Peštera 15) - grotte près du v.Krušuna, au lieu-dit Beltinski dol. Longueur totale $101 \mathrm{~m}$ : Atheta macroptera

*Lv 73. Graždenica - grotte près du v.Černi Vit. Très humide, longue de $61 \mathrm{~m}$, un ruisseau la parcourt et sort à son entrée. Au fond de la galerie une cascade: Choleva angustata

*Lv 74. Propast 135 - gouffre près du Karlukovo, au lieu dit Gligore. Dénivellation $-21 \mathrm{~m}$ : Collembola

*Lv 75. Propast 136 - gouffre près de la gare Karlukovo, au lieu-dit Gligore (Grigora). Un puits, profond de $-17 \mathrm{~m}$ : Queedius mesomelinus skoraszewskyi

*Lv 76. Borova dupka 1 - gouffre près du v.Černi Osâm, au lieu-dit Borovo dere. Très humide, puits d'une dénivellation de $108 \mathrm{~m}$. Quelques flaques: Collembola

* Lv 77. Borova dupka 2 - gouffre près du v.Černi Osâm, au lieu dit Borovo dere: Rhinolophus hipposideros

* Lv 78. Izvora - grotte près du v.Polaten. Un ruisseau sort de son entrée. Par un orifice étroit on peu pénétrer dans le couloir et le suivre sur une dizaine de $\mathrm{m}$ : Sphaeromides polateni

*Lv 79. Imaneto - grotte près de la gare Karlukovo. Humide, longue 270 m, dénivellation $-10 \mathrm{~m}$ : Nesticus cellulanus

* Lv 80. Propast 112 - gouffre près de la gare Karlukovo, au lieu dit Gligore. Dénivellation -32 m. Sèche: Nesticus cellulanus 


\section{Cavités dans le district de Mihajlovgrad}

Mg 1. Mišin kamik - grotte à 2 km NW du v.Gorna Luka: Trichoniscus anophthalmus; Meta menardi

Mg 2. Levica vreloto - grotte près du v.Prevala: Hyloniscus riparius; Meta merianae; Nargus badius

Mg 3. Vreloto -- grotte près du v.Prevala: Oxychilus glaber striarius; Nopoiulus venustus

Mg 7. Jamata - grotte près du v.Gorna Luka: Meta menardi

Mg 8. Peč - grotte près du v.Gorna Luka: Meta menardi; Philonthus cephalotes, Quedius mesomelinus skoraszewskyi

Mg 11. Mitrevska dupka - grotte près du v.Mitrevci: Nesticus cellulanus; Aleochara diversa, Gnathoncu: rotundatus

Mg 12. Grimnena dupka - grotte près du v.Čerkaski (entre Čerkaski et Draganica): Lepthyphantes sofianus, Porrhomma convexum; Rhinolophus hipposideros, $R h$. blasii, Miniopterus schreibersi, Myotis myotis, M.capaccinii

*Mg 13. Šokjovec - grotte près du v.Čerkaski: Serboiulus spelaeophilus

* Mg 14. Mramornata peštera - grotte près de Berkovica, dans la carrière Begovica. Très humide. Actuellement démolie: Serboiulus spelaeophilus

* Mg 15. Ajdučkata (Hajduškata) peštera - grotte-gouffre près du v.Prevala. Longueur $70 \mathrm{~m}$, dénivellation -36 m: Trachysphaera costata

*Mg 16. Dupkata - grotte-gouffre près du v.Gorna Verenica. Longueur $38 \mathrm{~m}$, dénivellation totale $-16 \mathrm{~m}$. Argile humide, quelques flaques et du bois pourri: Oxychilus glaber siriarius, Daudebardia rufa;Polydesmus renschi; Lepthyphantes spelaeorum, Meta menardi; Quedius fulgidus, Pristonychus punctatus; Rhinolophus hipposideros

*Mg 17. Drânkalna dupka - gouffre près du v.Dolno Ozirovo, au lieu dit Krešta. Puits humide, profond de $74 \mathrm{~m}$. Quelques flaques: Porrhomma convexum

*Mg 18. Grebenjo -- gouffre près du v.Dolno Ozirovo, au lieu-dit Grebenjo. Partie horizontale argileuse, longue d'une dizaine de $\mathrm{m}$, et un gouffre peu humide. Dénivellation totale d'env. 20 m: Lithobius lakatnicensis; Protoleptoneta bulgarica; Rambousekiella ledenicensis

*Mg 19. Nikolova jama - grotte près du v.Dolna Ozirovo: Nesticus cellulanus; Typhloiulus bureschi

*Mg 20. Vodnata dupka - grotte près du v.Dolno Ozirovo: Scoliopteryx libatrix

* Mg 21. Veneco 2-Srednjata - grotte près du v.Čeljustnica. Longueur $19 \mathrm{~m}$ : Oxychilus glaber striarius; Cyphoniscellus gueorguievi; A theta macroptera

*Mg 22. Veneco 1-Gornjata - grotte près du v.Čeljustnica. Longueur $33 \mathrm{~m}$ : Oxychilus glaber striarius; Hoplopholcus forskali; Triphosa sabaudiata

*Mg 23. Micina dupka - grotte près du v.Prevala. Longueur $18 \mathrm{~m}$ : Polydesmus renschi

\section{Cavités dans le district de Pazardžik $-\mathbf{P z}$}

Pz 1. Lepenica - grotte près de Velingrad: Philopotamus montanus 
Pz 3. Pešterata - grotte près de la gare Belovo: Quedius mesomelinus skoraszewskyi $\mathrm{Pz}$ 4. Novata peštera - grotte près de la ville de Peštera: Bulgaronethes haplophtalmoides, Hyloniscus riparius; Pseudacherontides spelaea; Drosophila fasciata, Helomyza captiosa

Pz 7. Dupčeto - grotte près de Velingrad. Lepthyphantes zimmermani

\section{Cavités dans le district de Pernik - Pk}

Pk 1. Duhlata - grotte près du v.Bosnek. Brachydesmus cristofer; Neotrombicula autumnalis, Odontoscirus iota, Cunaxoides croceus; Onychiurus fimetarius, Arrhopalites pygmaeus; Quedius mesomelinus; Heteromyza? rotundicornis, Triphleba antricola; Rhinolophus euryale

Pk 2. Živata voda - grotte dans la montagne Vitoša. Nesticus cellulanus; Quedius mesomelinus skoraszewskyi

Pk 4. Filipovskata peštera - grotte près du v.Filipovci: Meta menardi

*Pk 7. Bezimenna peštera - grotte près de Trân, dans le défilé de la rivière Erma.: Meta menardi

*Pk 8. Černa padina - grotte près de la ville Trân: Meta menardi

*Pk 9. Jamkata - gouffre près du v.Rajanci. Deux orifices donnent accès dans un gouffre presque clair, avec quelques embranchements: Rhinolophus blasii

*Pk 10. Dimova jamka - grotte près du v.Smirov dol. Longueur env. $30 \mathrm{~m}$, dénivellation env. $15 \mathrm{~m}$, deux entrées: Micropterna nycterobia; Rhinolophus ferrumequinum

*Pk 11. Goljamata peštera - grotte près du v.Gorna Vrabča: Atheta macroptera

\section{Cavités dans le district de Pleven - Pn}

Pn 1. Kajalâškata peštera - grotte près de Pleven. Aménagée: Thelida atricornis Pn 2. Hajduškata peštera - grotte près du v.Devenci: Trichoniscus bureschi; Trachysphaera costata; Quedius mesomelinus skoraszewskyi; Liancalus virens

Pn 3. (No nouveau au lieu de Vr 41). Temnata dupka - grotte près du v.Reselec: Trichoniscus pusillus pusillus

*Pn 4. Sedlarkata - grotte près du v.Rakita. Une galerie, longue de $1214 \mathrm{~m}$, dont le sol est inondé: Trachysphaera costata, Nopoiulus venustus; Trechus quadristriatus, Quedius mesomelinus skoraszewskyi

*Pn 5. Genina peštera - grotte près du v.Sadovec, au lieu-dit Studenec, sur la rive gauche de la rivière Vit. Humide, une large entrée, beaucoup de guano: Miniopterus schreibersi

*Pn 6. Gornoto ezero - grotte près du v.Breste. Une galerie sans embranchements, longue de $50 \mathrm{~m}$. Ruisseau, qui sort à l'entrée: Niphargus bureschi; Nesticus cellulanus, Meta menardi

Pn 7. Nanin kamâk - grotte près'du v.Muselievo, au lieu-dit Nanin kamâk. Longueur totale $230 \mathrm{~m}$. Beaucoup de guano: Rhinolophus mehelyi, Myotis myotis, M.bly thi oxygnathus, M.capaccinii, Miniopterus schreibersi 


\section{Cavités dans le district de Plovdiv - Pv}

Pv 1. Han maara - grotte près du châlet Raj, dans le Balkan Central. Récemment a été découvert un étage inférieur, dont le sol est presque recouvert d'un ruisseau: Trachelipus balticus; Tegenaria silvestris, Porrhomma convexum; Lithobius agilis

Pv 4. Topčika - grotte près du v.Dobrostan: Balkanopetalum beshkovi; Monotarsobius crassipes; Quedius mesomelinus skoraszewskyi

Pv 5. Ledenicata - gouffre près du v.Dobrostan, au lieu dit Vodite: Oxychilus glaber striarius

Pv 7. Gargina dupka (nom correct au lieu de "Garvanica") - grotte près du v.Mostovo: Meta merianae; A theta macroptera

Pv 8. Jamata - grotte près du v.Dobrostan: Balkanopetalum rhodopinum; Monotarsobius crassipes; Meta menardi; Pristonychus punctatus

Pv 9. Erkjuprija - grotte près du v.Mostovo: Oxychilus glaber striarius, Daudebardia wiktori;Porrhomma convexum

Pv 10. Hralup (Hralupa) - grotte près du v.Dobrostan: Balkanopetalum rhodopinum; Thracophilus beroni; Lepthyphantes leprosus, Meta menardi

Pv 11. Ledenicata - grotte près des ponts naturels "Čudnite mostove", Rhodopes: Atheta macroptera

*Pv 12. Cânkaliva dupka - gouffre près de la ville Assenovgrad, au lieu-dit Kuru dere. Puits d'une profondeur de $25 \mathrm{~m}$, plusieurs pierres au fond: Trichoniscus bureschi; Atheta mucroptera, Pristonychus punctatus

*Pv 13. Fidjafkinata dupka - grotte près du v.Izbegli. Une galerie argileuse, longue d'une vingtaine de m: Scutigera coleoptrata; Lepthyphantes insignis; Pristonychus punctatus, Langelaridia sp.

*Pv 14. Ivanova voda - gouffre près du v.Dobrostan. Dénivellation totale $131 \mathrm{~m}$. Très humide, un lac vers le fond: Chondrula sp. (murotraga? ), Xerophila aberrans, Clausilia sp.; Petroeus (Ena); Niphargus sp. Lepthyphantes centromeroides, Porrhomma convexum, Porrhomma sp.; Rhagidia spelaea, Rh.wolmsdorfensis; Eupauropus ef. cycliger; Isotomiella minor, Tomocerus sp. Unidentatus, Lepidocyrtus sp., Orchesella sp., Neelidae gen.sp.; Tachys sp., Atheta trinotata, ? Philonthus sp; Speolepta leptogaster, Trichocera regelationis, Limosina ? racovitzai, Eccoptomera obscura, Limnobia nubeculosa, Penicillidia dufouri; Myotis myotis, M. blythi oxygnathus

*Pv 15. Hajduškata dupka - grotte près du v.Dobrostan: Lepthyphantes leprosus *Pv 16. Ahmetjova dupka - gouffre près du v.Dobrostan. Dénivellation - 13 m., une salle concrétionnée, ay ant des dimensions $26 \times 13 \mathrm{~m}$ : Atheta macroptera

*Pv 17. Julen ere 2 - grotte près du v.Hristo Danovo, au lieu-dit Julen ere (Stara planina). Cette petite grotte ce trouve sur le bord d'une route forestière récemment creusée. Plusieurs stalagmites et quelques flaques: Trichoniscus bulgaricus; Trachysphaera costata, Polydesmus complanatus illyricus

\section{Cavités dans le district de Razgrad - Rz}

Rk 2. Mokrata peštera - grotte près du v.Topčii: Hyloniscus riparius, Cylisticus 
convexus; Tegenaria domestica, Meta menardi, Nesticus cellulanus

$\mathbf{R z}$ 3. Kulina dupka - grotte près du v.Krivnja: Lepthyphantes leprosus; Atheta macroptera

*Rz 4. Kaca peštera - grotte près du v.Seslav. Labyrinthe d'une longueur totale de 90 m: Trachelipus rathkei; Lepthyphantes leprosus, Meta menardi, Nesticus cellulanus; Pristonychus cimmeriuss,

\section{Cavités dans le district de Ruse (Roussé) - Rs}

Rs 1. Orlova čuka - grotte près du v.Pepelina. Corr.: longueur totale des galeries explorées plus de $10 \mathrm{~km}$ (la plus longue grotte connue en Bulgarie): Lepthyphantes insignis; Trechus austriacus, T.subnonatus, T.cardioderus balcanicus, Quedius mesomelinus skoraszewskyi, Mycetaea hirta

Rs 3. Tâmnata peštera - grotte près du v.Tabačka: Trichoniscus anophthalmus intermedius

*Rs 4. Propastta - gouffre près du v.Pepelina: Lepthyphantes leprosus

*Rs 5. Dil Nikolca - grotte près du v.Pepelina: Tegenaria domestica

\section{Cavités dans le district de Sliven - SI}

Sl 1. Dolnata maaza - grotte près du v.Bjala: Atheta macroptera; Scoliocentra caesia

Sl 3. Gornata maaza - grotte près du v.Bjala: Oxychilus depressus

SI 4. Lednicata - grotte-gouffre à $13 \mathrm{~km}$ du v.Medven: Lithobius mutabilis

Sl 6. Kipilovskata peštera - grotte près du v.Kipilovo: Antrodicus falcatus; Stenophylax mitis

Sl 7. Niric - gouffre près de Kotel: Hyloniscus riparius

Sl 8. Orlovata peštera - grotte près de Kotel: Meta menardi

S1 9. Drjanovskata peštera - grotte près de Kotel: Bilobella digitata

Sl 11. Meča dupka - grotte près de Kotel: Ligidium herzegowinense; Meta menardi Sl 12. Lednika - gouffre-grotte près de Kotel. Corr.: dénivellation totale $242 \mathrm{~m}$, longueur totale $1111 \mathrm{~m}$ : Atheta macroptera, Trechus cardioderus balcanicus

Sl 15. Zmejovi dupki - grotte près de Sliven: Medon fusculus, Ocypus globulifer, Quedius fulgidus, Qu.obliteratus

Sl 16. Starganakovoto - gouffre près de Kotel: Nesticus cellulanus

Sl 17. Dimova dupka - grotte près de Kotel: Meta menardi

Sl 18. Subattá - grotte près de Kotel: Atheta macroptera, Aleochara diversa

*SI 20. Mâglivata peštera - grotte près de Kotel. Longueur 136 m, dénivellation -48 m. Argile: Polydesmus denticulatus beroni, Typhloiulus kotelensis

*SI 21. Nehrov trap - grotte-gouffre près du v.Kipilovo. Longueur 68 m, dénivellation -31 m. Eboulis, guano: Lepthyphantes leprosus, Meta segmentata, Nesticus cellulanus

*Sl 22. Žilovata češma - grotte près de Kotel, au dessus de la fontaine portant le même nom. Etroite, humide: Quedius mesomelinus skoraszewskyi;

SI 23. Čerešata (Drânči dupka) gouffre près de Kotel, au lieu-dit Zlosten. Humide, 
profondeur env. $50 \mathrm{~m}$ : Stenophylax vibex speluncarum

*Sl 24. Manolovata peštera - grotte près de Kotel. Longueur $120 \mathrm{~m}$. Se termine par un siphon: Trechus crucifer; Micropterna sequax

*Sl 25. Kozjata peštera - grotte près du v.Kipilovo, à $12 \mathrm{~km} \mathrm{SW}$ du village. Une large galerie, longue d'env. $60 \mathrm{~m}$, haute 10-15 m: Quedius mesomelinus skoraszewskyi

\section{Cavités dans le district de Smoljan - Sm}

Sm 1. Lednicata - grotte près du v.Gela, au lieu dit Kekeza. Alt. 1538 m, longueur totale $1419 \mathrm{~m}$ : Barbastella barbastellus, Plecotus auritus, Eptesicus serotinus, Myotis myotis, M.blythi oxygnathus, M.emarginatus, M.mystacinus, M.brandti; Micropterna sequax:

Sm 3. Dupkata - grotte près du v.Progled. au lieu dit Rožen: Helomyza captiosa, Scoliocentra caesia, Eccoptomera emarginata

Sm 4. Sbirkovata peštera (jadis Pešterata, in Guéorguiev et Beron, 1962) - grotte près du v.Progled: Helomyza captiosa Myotis mystacinus

Sm 5. Samurskata dupka (jadis Jamata, in Guéorguiev et Beron, 1962) - grotte près de Čepelare: Plecotus auritus, Myotis mystacinus

Sm 6. Čelečkata peštera (Čoveškata peštera) - grotte près du v.Orehovo: Bulgarosoma tridentifer; Lithobius lakatnicensis; Helomyza captiosa

Sm 10. Vaklite dupki - grotte près du v.Zabârdo: Oxychilus glaber striarius; Meta menardi

*Sm 13. Imamova dupka - grotte près du v.Jagodina. Plusieurs galeries d'une longueur totale de 7000 m: Trichoniscus raitshevi; Amaurobius claustrarius, A.c. balcanicus, Meta menardi, Nesticus cellulanus; Bulgarosoma meridionale; Myotis bechsteini, M.emarginatus, Eptesicus serotinus

*Sm 14. Tâmnicata - grotte près du v.Kremene, quartier Poprelka: Rhinolophus hipposideros, Myotis myotis

*Sm 15. Nadarskata peštera - grotte près du v.Kremene, Quartier Nadarci: Trichoniscus rhodopiense

*Sm 16. Boevskata peštera - grotte près du v.Boevo. Longueur de l'axe principale $35 \mathrm{~m}$, largeur max. 2,4 m. haute jusqu'à $3 \mathrm{~m}$ : Trichoniscus rhodopiense; Lepthyphantes centromeroides, L.leprosus, Centromerus jacksoni, Tegenaria silvestris; Atheta macroptera

*Sm 17. Borovskata peštera - grotte près du v.Borovo: Meta menardi

*Sm 18. Sančova dupka - grotte près du v.Jagodina. Longueur de l'axe principale $455 \mathrm{~m}$, longueur totale $888 \mathrm{~m}$, haute jusqu'à $13 \mathrm{~m}$, large jusqu'à $47 \mathrm{~m}$ : Lepthyphantes centromeroides, Meta menardi, Nesticus cellulanus; Stenophylax vibex speluncarum, Micropterna sequax; Myotis myotis, M.emarginatus, Eptesicus serotinus

*Sm 19. Dolna Karanska dupka - grotte près du v.Jagodina: Lepthyphantes centromeroides; Chromatoiulus rhodopinus; Myotis myotis

*Sm 20. Gorna Karanska dupka - grotte près du v.Jagodina. Alt. 1065 m: Elaphoidella angelovi; Tegenaria ferruginea, Meta menardi; Myotis emarginatus, M.nattereri 
*Sm 21. Ramadanovata dupka - grotte près du v.Jagodina: Meta menardi

*Sm 22. Meča dupka - grotte près de Pamporovo. Humide, plusieurs gours. Longueur totale 108 m. Alt.1560 m. Dénivellation -16 m: Meta menardi, Antrohyphantes rodopicus; Micropterna inycterobia

*Sm 23. Rizovica - grotte près du v.Kremene, quartier Poprelka. Longueur totale 112 m. Deux entrées: Lithobius lakatnicensis; Lepthyphantes rhodopensis; Atheta macroptera

*Sm 24. Handâka - grotte près du v.Mogilica, quartier Uhlovica: Lepthyphantes centromeroides; Quedius mesomelinus skoraszewskyi

*Sm 25. Malkata peštera - grotte près du v.Borikovo: Meta menardi; Trirhacus sp.; Troglophilus $s p$.

*Sm 26. Červena dupka - grotte près du v.Borikovo. Longueur totale $440 \mathrm{~m}$, haute jusqu'à $8 \mathrm{~m}$. Argile humide: Troglophilus sp.; Myotis myotis

*Sm 27. Golobovica 1 (Goloboica 1) - grotte près du v.Košnica. La plus haute des 3 grottes superposées: Diptera

*Sm 28. Golobovica 2 - grotte près du v.Košnica, au lieu-dit Garga deré. Une grande salle, dont le sol est recouvert de guano: Rhinolophus ferrumequinum

*Sm 29. Golobovica 3 - grotte près du v.Košnica. Le sol de cette grotte qui est la plus basse des trois "Golobovica" du complexe, est submergé par un ruisseau. On peut pénétrer en barque $60 \mathrm{~m}$. La grotte communique avec Golobovica 2: Micropterna fissa

*Sm 30. Garvanjovica - grotte près du v.Turen (quartier Poprelka), sur la rive gauche de la rivière Garga dere. Longue $70 \mathrm{~m}$, un ruisseau forme dans la grotte 3 lacs, dont le troisième profond plus de $3 \mathrm{~m}$ : Balkanopetalum rhodopinum; Trirhacus sp.

${ }^{*}$ Sm 31. Ulcata - grotte près du v.Mogilica, quartier Uhlovica: Rhinolophus ferrumequinum

*Sm 32.Djavolskoto gârlo - gouffre près du v.Trigrad. Une rivière entre par l'entrée supérieure et sort $102 \mathrm{~m}$ plus bas, en formant un siphon et quelques lacs. Plusieurs troncs de pins, entraînés par l'eau. Le gouffre est très humide: Chromatoiulus beroni; Nesticus cellulanus; Trechus subnotatus; Hydropsyche instabilis

\section{Grottes dans le district de Sofia - Sf}

Sf 3. Dinevata pešt - grotte près du v.Ginci: Centromerus jacksoni, Meta menardi; Stenophylax vibex speluncarum, S. mitis, Micropterna nycterobia, M. sequax

Sf 4. Svetata voda - grotte près du v.Ginci: Helomyza captiosa

Sf 5. Krivata pešt - grotte près du v.Ginci: Acantholeria cineraria; Micropterna nycterobia

Sf 7. Peštta (Bučinska pešt) - grotte près du v.Bučino prohod: Tegenaria silvestris, Meta merianae

Sf 8. Temnata dupka - grotte près du v.Berende Izvor: Brachydesmus herzegowinensis confinis; Quedius mesomelinus skoraszewskyi, A theta macroptera

Sf 9. Peruna dupka - grotte près du v.Kalotina: Meta menardi

Sf 12. Kolibata - grotte près du v.Beledie han: Trachelipus balticus; Chiromyia flava, Thelida atricornis (?) 
Sf 15. Jamata - grotte près de la gare Cerovo: Meta menardi

Sf 16. Otečestvo - grotte-mine près du v.Iskrec: Quedius mesomelinus skoraszewskyi

Sf 22. Golaškata peštera - grotte-mine près du v.Golak: Thelida atricornis

Sf 24. Mečata dupka - grotte près du v.Želen: Typhloiulus bureschi; Meta merianae, M.menardi; Duvalius pretneri

Sf 25. Javoreckata peštera - grotte près du v.Lakatnik: Lepthyphantes centromeroides; Helomyza captiosa

Sf 26. Svârdelo - gouffre près de Lakatnik: Trachysphaera orghidani; Micropterna nycterobia

Sf 29. Zidanka - grotte près de la gare Lakatnik: Quedius mesomelinus skoraszewskyi

Sf 30. Temnata dupka - grotte près de la gare Lakatnik: Quedius mesomelinus skoraszewskyi, Micrambe translatus, Cryptophagus scutellatus; Scoliocentra brachypterna, Drosophila fasciata; Acrolepia granitella, A.pulicariae, Antophila limbata, Scoliopteryx libatrix, Triphosa dubitata

Sf 32. Ražiška dupka - grotte près de la gare Lakatnik (autre nom: Suhata pestera): Micrambe translatus, Cryptophagus scutellatus; Nycteridopsylla ancryluris johanae; Limosina racovitzai; Antophila limbata; Eptesicus serotinus

Sf 33. Svinskata dupka - grotte près de la gare Lakatnik; Meta menardi

Sf 34. Kozarskata peštera - grotte près de la gare Lakatnik: Trachysphaera orghidani; Acrolepia pulicariae

Sf 36. Sedmovratica - grotte près de la gare Lakatnik: Armadillidium klugi

Sf 38. Kitova kukla - grotte près du v.Druževo: Meta menardi; Stenophylax vibex speluncarum, S.mitis, Micropterna nycterobia

Sf 40. Jamata - gouffre près du v.Lakatnik: Oxychilus glaber striarius

Sf 41. Čavkite - gouffre près du v.Milanovo: Ceratophysella armata; Drosophila fasciata

Sf 44. Suhata jama - grotte-gouffre près du v.Druževo: Meta menardi

Sf 47. Vodnata pešt - grotte près du v.Lipnica: Meta menardi; Micropterna nycterobia, Mesophylax aspersus

Sf 52. Vodnata peštera - grotte près de la gare Cerovo: Helomyza captiosa

Sf 57. Porojnata dupka - gouffre-grotte près du v.Zasele: Quedius mesomelinus

Sf 59. Radjova propast - gouffre près du v.Milanovo: Centromerus lakatnikensis

Sf 60. Gornata dupka - grotte près de la gare Cerovo: Hyloniscus riparius

Sf 62. Zahlupena dupka - grotte près de la ville Etropole: Quedius troglophilus

*Sf 67. Obija den - gouffre près d'Etropolé. Dimensions de l'orifice $4 \times 1 \mathrm{~m}$. Dénivellation totale $29 \mathrm{~m}$. Fond sableux: Pseudoscorpionida

*Sf 68. Svinaka - grotte près du v.Leskov dol: Lepthyphantes centromeroides

*Sf 69. Hajduška dupka - grotte près du v.Beledié han: Meta menardi; Atheta macroptera

*Sf 70. Meča dupka - grotte - gouffre près du v.Meča poljana. Entrée triangulaire, large de $1,5 \mathrm{~m}$ et haute d'env. $6 \mathrm{~m}$. Longueur totale $142 \mathrm{~m}$, dénivellation totale -53 m. Argile, quelques gours. Alt. 1150 m: Meta menardi; Onychiurus ghidinii

*Sf 71. Pepina dupka - grotte près du v.Ginci. Longueur 29 m: Meta menardi 
*Sf 72. Zdraveška dupka - gouffre près du v.Druževo. Dénivellation $-12 \mathrm{~m}$. Beaucoup de mousses sur les parois: Meta menardi

*Sf 73. Vâlči kamâk - grotte près du v.Dragoil: Tegenaria domestica

*Sf 74. Padeż - gouffre près du v.Breze. Alt. $1020 \mathrm{~m}$., dénivellation $47 \mathrm{~m}$, plusieurs nids de Pyrrhocorax graculus sur la première terrasse: Lytopelte bureschi, Vitrea diaphana; Lepthyphantes sofianus; Lithobius erythrocephalus (? ); Serboiulus spelaeophilus; Quedius mesomelinus skoraszewskyi, Atheta macroptera; Pyrrhocorax graculus

*Sf 75. Blagova dupka 1 - gouffre près d'Etropole, au lieu dit Blagovoto. Profondeur $-110 \mathrm{~m}$. Au fond accumulations d'argile humide: Porrhomma convexum *Sf 76. Paraklisa - grotte près de la gare Bov, sur la rive gauche de l'Iskâr. A deux étages, longueur totale $41 \mathrm{~m}$, longueur de l'axe principale $30 \mathrm{~m}$. Humide, plusieurs stalactites. Entrée 8 × $5 \mathrm{~m}$ : Typhloiulus bureschi, Balkanopetalum armatum

Sf ? Petite grotte près de Razboiški monastir: Meta menardi

Sf ? Petite grotte près du v. Lopušna, au lieu dit Mogilo: Meta menardi

Sf ? Petit gouffre près du v.Lopušna, au lieu dit Mogilo: Meta menardi, Pardosa hortensis, Amaurobius pallidus

\section{Cavités dans le district de Stara Zagora $-\mathrm{Sz}$}

Sz 3. Toplata dupka - grotte près de la gare Boruštica: Oxychilus depressus; Meta menardi; Micropterna malaspina

\section{Cavités dans le district de S̉umen - Šn}

*Šn 1. Katakombite - grotte près de Šumen. Autre nom: Labirinta. Trois grottes superposées, d'une longueur totale de $85 \mathrm{~m}$ : Lepthyphantes leprosus, Meta menardi *Šn 2. Arkovna - grotte près de Šumen: Nesticus eremita, Meta menardi

*Šn 3. Peštera 5 - grotte près de Šumen, en dessus du châlet des spéléologues. Sèche, longueur 29 m: Tegenaria silvestris

*Šn 4. Dipsiskoju - gouffre-grotte près de Šumen. Longueur $98 \mathrm{~m}$, quelques concrétions: Trachysphaera costata; Atheta macroptera; Micropterna malaspina

*Šn 5. Žâlâd - grotte près de Šumen: Meta menardi, Nesticus cellulanus

*Šn 6. Zandana - grotte près de Šumen. Deux étages, longueur totale $1800 \mathrm{~m}$, un ruisseau y coule: Stenophylax vibex speluncarum

*Šn 7. Peštera 1 - grotte près de Sumen. Une galerie humide, longue de $29 \mathrm{~m}$. Située près de la route Šmen-le châlet "K.Avramov": Micropterna nycterobia; Quedius mesomelinus skoraszewskyi

*Šn 8. Lozevskata peふtera .- grotte près de Sumen, vers le village Lozevo. Longueur $87 \mathrm{~m}$, dénivellation $5 \mathrm{~m}$ : Oxychilus glaber striarius; Nesticus cellulanus; Atheta macroptera

*Šn 9. Krumovi porti - grotte près de Sumen. Complèxe de plusieurs petites grottes d'une longueur totale de 179 m. Sèche: Pristonychus punctatus; Rhinolophus ferrumequinum 


\section{Cavités dans le district de Târgovišste $-\mathrm{Tg}$}

Tg 2. Prolazkata peštera - gouffre-grotte près du v.Prolaz: Polydesmus denticulatus

\section{Cavités dans le district de Veliko Târnovo - Tn}

Tn 2. Kalugerova dupka - grotte près du v.Arbanasi: Hyloniscus riparius; Microchordeuma sp.

Tn 3. Golema Podlisca - grotte près du v.Beljakovec: Trichoniscus tenebrarum; Helomyza captiosa

Tn 6. Trošana - grotte près de Veliko Târnovo: Helomyza serrata

*Tn 10. Draganovskata peštera - grotte près du v.Draganovci: Longueur $45 \mathrm{~m}$, dénivellation $+9 \mathrm{~m}$. Un ruisseau y coule: Tegenaria silvestris, Meta menardi, Nesticus cellulanus, Atheta macroptera

*Tn 11. Jamata - grotte près du v.Draganovci. Longueur $11 \mathrm{~m}$ : Meta menardi

\section{Cavités dans le district de Vidin - Vd}

Vd 1. Vârkan (nom plus correct que "Vrkan") - grotte près du v.Družba (jadis Sv.Petâr). Corr.: lcngueur de l'axe principal $800 \mathrm{~m}$, dénivellation $-40 \mathrm{~m}$. Plusieurs Chauves-souris et grands amas de guano. Un ruisseau suit toute la longueur de la galerie, en formant un siphon au fond: Oxychilus glaber striarius; Trichoniscus bononiensis; Nesticus cellulanus; Miniopterus schreibersi

Vd 2. Margura - grotte près du v.Rabiša: Lepthyphantes centromeroides; Quedius mesomelinus skoraszewskyi

Vd 3. Cankinoto vrelo - grotte dans le village Granitovo: Agabus guttatus

Vd 4. Neprivetlivata (= Gornata propast sensu Guéorguiev \& Beron, 1962 = Prelaz sensu Beron \& Guéorguiev, 1967) - gouffre près de Belogradčik: Polydesmus renschi, Brachydesmus dadayi brusenicus; Quedius mesomelinus skoraszewskyi

Vd 7. Propast - gouffre-grotte près de la gare Orešec. Corr.: longueur totale $245 \mathrm{~m}$. Dénivellation totale $62 \mathrm{~m}$. Beaucoup de guano. La salle terminale a son sol occupé par un lac d'eau stagnante: Trichoniscus bononiensis; Heteromurus nitidus; Miniopterus scheibersi, Myotis myotis

Vd 8. Suhi peč - grotte près du v.Orešec. Longueur $192 \mathrm{~m}$ : Trichoniscus bononiensis; Lepthyphantes spelaeorum; Heteromurus nitidus; Quedius mesomelinus skoraszewskyi; Myotis capaccinii, M. blythi oxygnathus, Rhinolophus ferrumequinum; Feilis silvestris

Vd 10. Tâmni peč - grotte près du v.Vârbovo: Lepthyphantes leprosus, L.spelaeorum, Nesticus cellulanus; Serboiulus spelaeophilus; Quedius mesomelinus skoraszewskyi, Atheta macroptera

Vd 11. Golemi peč - grotte près du v.Vârbovo: Niphargus tauri pecarensis, N.bureschi; Lepthyphantes leprosus, Tegenaria domestica

Vd 14. Levi suhi peč - grotte près du v.Dolni Lom: Cyphoniscellus gueorguievi; Quedius mesomelinus skoraszewskyi; Rhinolophus ferrumequinum

Vd 15. Vodni peč - grotte près du v.Dolni Lom: Serboiulus spelaeophilus 
Vd 16. Tâmna dupka - grotte près du v.Târgovište. Longueur de l'axe principale $59 \mathrm{~m}$, longueur totale $120 \mathrm{~m}$. Périodiquement inondée: Trachysphaera orghidani, Brachydesmus dadayi, Serboiulus spelaeophilus; Quedius mesomelinus skoraszewskyi

*Vd 17. Elenova dupka - gouffre près du v.Bela, au lieu dit Džamiite. Longueur $22 \mathrm{~m}$, dénivellation $8 \mathrm{~m}$; argile humide: Hyloniscus flammula; Nesticus cellulanus

*Vd 18. Studena - grotte près du v.Vojnica. Longueur $203 \mathrm{~m}$. Une galerie basse, s'élargissant en salle avec beaucoup de guano et d'argile humide. Un ruisseau coule dans la grotte et sort à l'entrée: Oxychilus glaber striarius; Hyloniscus flammula; Nesticus cellulanus; Miniopterus schreibersi, Myotis myotis, M.blythi oxygnathus *Vd 19. Redaka 1-Gornata - gouffre près du v.Salaš. Longueur 97 m, dénivellation -20 m: Trichoniscus bononiensis; Serboiulus spelaeophilus; Choleva paskoviensis

*Vd 20. Mečata dupka - gouffre près du v.Salaš, au lieu dit Meškovica. Au fond du bois pourri: Trichoniscus bononiensis; Lepthyphantes spelaeorum, Centromerus jacksoni; Stenophylax mitis

*Vd 21. Prelaz - grotte près du v.Salaš, au lieu dit Ruškovica (ne pas confondre avec Vd 4 in Beron \& Guéorguiev, 1967 - ex errore). Plusieurs galeries d'une longueur totale de plus de $400 \mathrm{~m}$. Argile humide, par endroit quelques flaques: Oxychilus glaber striarius; Trichoniscus bononiensis; Micropterna nycterobia

*Vd 22. Kračimirskoto vrelo - grotte près du v.Stakevci. Longueur $147 \mathrm{~m}$, le sol est submergé par une rivière, profonde jusqu'à $3 \mathrm{~m}$. On y pénètre en barque: Trichoniscus bononiensis; Lepthyphantes leprosus, Araneus bituberculatus, Tegenaria ferruginea, Meta menardi, M.merianae; Rhinolophus ferrumequinum, Miniopterus schreibersi

*Vd 23. Studeni vrâh - grotte près du v.Vârbovo: Meta menardi

*Vd 24. Spuštenica - gouffre près du v.Vârbovo. Sèche, longueur $35 \mathrm{~m}$, dénivellation $-7 \mathrm{~m}$ : Meta menardi

*Vr 25. Vreloto - grotte près du v.Dolni Lom. Longueur $52 \mathrm{~m}$, dénivellation $-5 \mathrm{~m}$. Un ruisseau la parcourt et sort à son entrée: Nesticus cellulanus

*Vd 26. Radenov kladenec - gouffre près du v. Salaš, au lieu-dit Čemeričnica, au fond de la vallée Ziljan dol. Prof. 7-8 m. Source: Amphipoda

*Vd 27. Jame 1 - gouffre près du v.Târgovište. Profondeur $42 \mathrm{~m}$ : Lepthyphantes leprosus

*Vd 28. Jame 2 - gouffre près du v.Târgovište. Profondeur 55 m. Araneida

*Vd 29. Jame 3 - gouffre près du v.Târgovište. Longueur $240 \mathrm{~m}$, dénivellation -55 m: Trichoniscus bononiensis; Serboiulus spelaeophilus; Lepthyphantes spelaeorum; Quedius mesomelinus skoraszewskyi, Pristonychus punctatus; Stenophylax mitis

*Vd 30. Peina dupka - gouffre près du v.Vârbovo. Dénivellation -55 m: Lepthyphantes spelaeorum

*Vd 31. Dragaličeva dupka - gouffre près du v.Vărbovo: Pholcus opilionoides

*Vd 32. Djado Stanov stol - grotte-gouffre près du v.Delejna. Longueur $29 \mathrm{~m}$, dénivellation -8 m: Nesticus cellulanus; Cryptophagus cellarius

*Vd 33. Koritska glama - grotte près du v.Salaš, au lieu-dit Koritska glama. Longueur 48 m., dénivellation -14 m. Argile: Oxychilus glaber striarius; Typhloiulus 
strictus; Quedius mesomelinus skoraszewskyi

*Vd 34. Redaka 2 - grotte près du v.Salaš, tout près de Vd 19. Longueur totale $60 \mathrm{~m}$, longueur de l'axe principal 29 m. Argile humide: Serboiulus spelaeophilus; Quedius mesomelinus skoraszewskyi

*Vd 35. Carevica - gouffre près du v.Salaš. Sèche, longueur 25 m, dénivellation $-17 \mathrm{~m}$ : Oxychilus glaber striarius; Nesticus cellulanus

*Vd 36. Kurtovata peštera - grotte près du v.Vodna, au lieu-dit Lavicite. Sèche, longue de $24 \mathrm{~m}$ : Coleoptera

*Vd 37. Magura 2 - grotte près du v.Rabiša. Longue d'env. $300 \mathrm{~m}$, mais actuellement effondrée: Scutigera coleoptrata; Trechus cardioderus balcanicus

*Vd 38. Stanišina dupka - grotte près du v.Repljana, sur la colline Gradište. Alt. $720 \mathrm{~m}$, longueur totale $40 \mathrm{~m}$ : Serboiulus spelaeophilus; Scoliopteryx libatrix, Triphosa sabaudiate!

*Vd 39. Zemna dupka - grotte-gouffre près du v.Repljana, au lieu dit Gusta padinka (la colline Gradište). Longueur totale $49 \mathrm{~m}$. Descendant en gradins, à la fin un petit gouffre. Alt. $760 \mathrm{~m}$ : Rhinolophus hipposideros

*Vd 40. Tatarska dupka - grotte près du v.Repljana, au lieu-dit Esenjako. Alt $630 \mathrm{~m}$. Longueur totale $92 \mathrm{~m}$. Concrétions stalagmitiques: Rhinolophus ferrumequinum, Rh. hipposideros

*Vd 41. Medžak dupka - grotte près du v.Repljana, au lieu dit Medžaka (Čarna glama). Alt. $580 \mathrm{~m}$. Longueur totale $79 \mathrm{~m}$, deux étages. Concrétions, argile humide: Serboiulus spelaeophilus; Rhinolophus hipposideros

*Vd 42. Golema dıpka - grotte près du v.Repljana. Longueur totale $155 \mathrm{~m}$, deux étages, guano, dans le couloir supérieur vers la fin un peu de bois pourri: Heteromurus nitidius quadriocellatus; Triphosa sabaudiata; Rhinolophus ferrumequinum, Myotis myotis; M.blythi oxygnathus

*Vd 43. Dupkata v Prosečeno - grotte près du v.Repljana, au lieu dit Zapadje (la colline Gradište). Alt. $780 \mathrm{~m}$, longueur totale $35 \mathrm{~m}$ : Triphosa sabaudiata

*Vd 44. Zmijskata propast - gouffre près du v.Kračimir, au lieu dit Čemeričnica (la vallée Ziljan dol). Dénivellation $48 \mathrm{~m}, 2$ puits $(30$ et $11 \mathrm{~m})$. Petite entrée $(80 \times 35 \mathrm{~cm})$. Humide, suintements d'eau: Trichoniscus bononiensis; Serboiulus spelaeophilus; Rhinolophus hipposideros

$* V d 45$. Veneca - grotte près du v.Orešec, dans le front de la carrière au lieu-dit Veneca. Entrée fermée par une porte de fer. Longueur totale $196 \mathrm{~m}$. Plusieurs concrétions stalagrnitiques, beaucoup d'argile humide vers la partie terminale. Quelques flaques: Bathysciinae

*Vd 46. Falkovska ta peštera - grotte-gouffre près du v.Falkovec, dans une carrière abandonnée au bord de la route Ružinci - Falkovec, au lieu-dit Pionerski lager. Alt. $110 \mathrm{~m}$, longueur totale $45 \mathrm{~m}$. Un ruisseau coule au fond. Argile, amas de bois pourri: Cyphoniscellus gueorguievi; Serboiulus spelaeophilus; Heteromurus nitidus; Lasius niger; Rhinolophus ferrumequinum, Myotis myotis

*Vd 47. Dupkata - grotte près du v.Janjovec, au lieu-dit Glamata (NNW du village). Alt $340 \mathrm{~m}$, longueur totale $24,5 \mathrm{~m}, 2$ étages. Argile humide, un peu de guano: Pseudoscorpionida

*Vd 48. Dupkite - grotte à 2 entrées près du v.Belo pole. Située au lieu-dit Dâlgoto 
vârvište (Čukarite), sur la rive droite de la rivière Lom. Sèche, très pauvre en faune: Scoliopteryx libatrix

*Vd 49. Mejuva dupka 1 - gouffre près du v.Belotinci, au lieu-dit Brešta. Située dans la forêt de Quercus, près de la route qui suit la ligne électrique. Argile humide: Rhinolophus ferrumequinum

*Vd 50. Zankovska peštera - grotte près du v.Belotinci, sur la rive droite de la rivière Krácmarska reka. Alt. $130 \mathrm{~m}$. Une salle avec quelques petits embranchements, le sol est recouvert de guano: Serboiulus spelaeophilus; Heteromurus nitidus quadriocellatus; Lasius brunneus

Vd 51. Dupkata v Glamata - grotte près du v.Vârbovo: Trichoniscus bureschi, Cyphoniscellus gueorguievi

\section{Cavités dans le district de Vraca - Vr}

Vr 1. Prilepnata peštera - grotte près du v.Liljače: Gnathoncus nannetensis, Atheta macroptera; Limosina racovitzai

Vr 2. Drašanskata peštera - grotte près du v.Drašan: Daudebardia rufa; Niphargus bureschi; Nesticus cellulanus

Vr 3. Studenata dupka - grotte près de la gare Cerepiš: Oxychilus glaber striarius; Hoplopholcus forskali, Tegenaria silvestris; Phortica variegata

Vr 5. Serapionovata peštera - grotte près de la gare Čerepiš: Quedius mesomelinus skoraszewskyi, Atheta macroptera

Vr 16. Reznjoveta - gouffre près de Vraca: Meta menardi; Quedius mesomelinus skoraszewskyi

Vr 17. Ledenika - grotte aménagée près de Vraca: Trichoniscus bureschi; Nesticus cellulanus, Meta menardi, Tegenaria silvestris, Lepthyphantes sofianus; Catops picipes, Quedius mesomelinus skoraszewskyi; Actina nitens, Eccoptomera emarginata

Vr 22. Ponora - grotte près du v.Čiren: Trichoniscus bureschi; Typhloiulus bureschi; Quedius mesomelinus skoraszewskyi

Vr 23. Golemata jama - gouffre près du v.Čelopek: Meta menardi; Quedius mesomelinus skoraszewskyi, Catops coracinus

Vr 25. Zmejova dupka - gouffre près du v.Bistrec: Meta menardi

Vr 28. Tigančeto - grotte près du v.Liljače: Nesticus cellulanus; Atheta macroptera

Vr 29. Sipo - gouffre près du v.Gorno Ozirovo: Lepthyphantes sofianus

Vr 30. Mladenovata peštera - grotte près du v.Čren: Atheta sulcifrons

Vr 31. Garvanec - gouffre près de Vraca: Bryaxis beroni, Quedius mesomelinus skoraszewskyi

Vr 32. Zmejova dupka II - gouffre près du v.Bistrec: Lepthyphantes sofianus

Vr 34. Dupna mogila - grotte près du v.Dolna Bešovica: Trachysphaera costata; Protoleptoneta bulgarica; Atheta macroptera

Vr 35. Propastta (Ledeniška jama) - gouffre près de Vraca: Oxychilus depressus

Vr 37. Govedarskata dupka - grotte près du v.Čiren: Trachysphaera costata

Vr 38. Bulina dupka - gouffre-grotte près du v.Čiren: Daudebardia brevipes;

Trechus quadristriatus, Atheta sulcifrons, Choleva agilis 
Vr 40. Kalnata dupka - gouffre près de Vraca: Ceratophysella armata; Quedius mesomelinus skoraszewskyi

Vr 41. Voir Pn 3.

Vr 42. Tošova dupka (nom correct au lieu de "Kalna Mâtnica") - grotte près du v.Glavaci (non pas de Beli Izvor): Cyphoniscellus bulgaricus, Trichoniscus anophthalmus; Lithobius lakatnicensis; Typhloiulus bureschi; Hoplopholcus forskali, Meta menardi, M.merianae, Nesticus cellulanus; Ceratophysella armata; Bryaxis beroni, Duvalius beroni, Quedius mesomelinus skoraszewskyi, Atheta macroptera, Mycetaea hirta

*Vr 44. Popovata peštera - grotte près du v.Gabare, au lieu-dit Vutkovskite vârtopi. Quatres entrées larges, 4 entrées étroites, plusieurs couloirs en labyrin the. Alt. 260 m, longueur totale 530 m: Niphargus bureschi, Niphargus sp.; Typhloiulus bureschi; Meta merianae, Nesticus cellulanus; Quedius mesomelinus; Leptocera sp., Limnobiidae, Culicidae, Helomyzidae

*Vr 45. Meča dupka - grotte près du v.Ljutadžik. Longueur 66 m, se termine par un lac: Meta merianae

*Vr 46. Dupkata - grotte près du v.Brusen: Meta segmentata

*Vr 47.. Lednicata - gouffre près de Vraca. L'entrée assez large de ce gouffre se trouve dans la forêt près de la fontaine "Barkite". Humide, profondeur $52 \mathrm{~m}$. Onychiurus subgranulosus, Pseudosinella duodecimocellata

* Vr 48. Ezeroto - grotte près du v.Gabare: Meta menardi

*Vr 49. Capkula -- gouffre près du v.Dârmanci. Sec, profond de $-25 \mathrm{~m}$. Grands éboulis: Meta menardi, Nesticus cellulanus

*Vr 50. Propast 8 - gouffre près de la gare Zverino. Puits d'une profondeur de -16 m non loin de Pancovi gramadi: Meta menardi

*Vr 51. Ohodenskata peštera - grotte près du v.Ohoden. Très humide, longue de $83 \mathrm{~m}$, plusieurs flaques. On y pénètre par un tunnel artificiel: Folsomia candida

*Vr 52. Sokolskata dupka - grotte près du v.Ljutadžik. Deux galeries d'une longueur totale de plus de $300 \mathrm{~m}$. Ruisseau, formant plusieurs lacs: Centromerus bulgarianus, Tegenaria silvestris, Meta menardi; Myotis myotis, M.blythi oxygnathus *Vr 53. Beljar - gouffre-grotte près de Vraca. Dénivellation $-240 \mathrm{~m}$. Large galerie descendante, un ruisseau la suit et se perd au fond. Plusieurs stalactites, argile humide, plusieurs flaques: Niphargus bureschi; Bureschia bulgarica, Sphaeromides bureschi; Typhloiulus staregai; Centromerus bulgarianus

*Vr 54. Briša - grotte près du v.Gabare: Hoplopholcus forskali, Tegenaria ferruginea

*Vr 55. Barkite 1 (Peštera 14) - grotte près de Vraca, au lieu-dit Barkite, près de la fontaine. Longueur totale $213 \mathrm{~m}$, dénivellation $65 \mathrm{~m}$. Humide, un ruisseau la parcourt. Plusieurs stalactites: Nesticus cellulanus, Lepthyphantes sofianus

*Vr 56. Propast 2 - gouffre près du v.Kunino: Lepthyphantes leprosus

*Vr 57. Starate prodânka - grotte-gouffre près du v.Gabare. Dimensions de l'entrée: largeur $0,5-1 \mathrm{~m}$, longueur $5 \mathrm{~m}$. Un ruisseau parcourt la grotte, on y trouve quelques lacs. Longueur totale $553 \mathrm{~m}$ : Niphargus bureschi; Meta menardi, Linyphiidae; Rhinolophu's ferrumequinum

*Vr 58. Moravata - grotte-gouffre près du v.Gabare, au lieu-dit de même nom. 
Humide, au fond il y a un petit lac. Longueur totale $67 \mathrm{~m}$, dénivellation $-40 \mathrm{~m}$ : Daudebardia sp.; Niphargus bureschi; Porrhomma sp.; Typhloiulus bureschi; Pseudophonus rufipes

*Vr 59. Jankulova dupka - grotte à $500 \mathrm{~m}$ du v.Gabare, sur la rive gauche de la rivière Gabarska reka. Longueur totale $73 \mathrm{~m}$ : Diptera

*Vr 60. Gâgla - gouffre près du v.Gabare (4 $\mathrm{km}$ à l'Est du village). L'entrée $(1,5 \times 3 \mathrm{~m})$. Alt. $280 \mathrm{~m}$ Profondeur $64 \mathrm{~m}$, dont $50 \mathrm{~m}$ en puits vertical: Niphargus sp.; Nesticus sp., Porrhomma sp.; Quedius mesomelinus skoraszewskyi, Atheta sulcifrons

*Vr 61. Čerešarskata propast - gouffre près du v.Ljutadžik, au lieu-dit Čerešarskata kukla. Profondeur env. 60 m, puits vertical. Au fond de l'argile humide: Diptera

*Vr 62. Popudžijnicata - gouffre près du v.ČCiren. Puits vertical, profond de $24 \mathrm{~m}$ : Quedius mesomelinus skoraszewskyi

*Vr 63. Govedarnika - grotte près du v.Liljače. Longueur $18 \mathrm{~m}$. Argile humide: Choleva paskoviensis, Ch.agilis, Ch.oblonga

*Vr 64. Šišmanovec - grotte près de la gare Čerepišs: Balkanopetalum armatum

*Vr 65. Vratnik - grotte près du v.Ljutadžik: Meta menardi, M.merianae, Tegenaria silvestris

*Vr 66. Kondžova kruša - grotte-gouffre près du v.Čiren. Un puits de $-18 \mathrm{~m}$ donne accès dans une grotte, longue de $552 \mathrm{~m}$, dont les galeries sont inondées par un ruisseau: Niphargus bureschi

*Vr 67. Zvânkova dupka - gouffre - grotte près du v.Liljače. Puits de $-19 \mathrm{~m}$ et galerie inondée de $38 \mathrm{~m}$ : Niphargus bureschi

*Vr 68. Pešketo - grotte-complexe karstique près du v.Liljače. Longueur totale $112 \mathrm{~m}$ : Trachelipus rathkei

*Vr 69. Pešova jama - grotte près du v.Čiren. Périodiquement inondée: Trechus quadristriatus

Razrušenata peštera - grotte près de la gare Kunino, dans la carrière, actuellement démolie. Très humide, plusieurs stalactites: Balkanoniscus corniculatus

\section{PUBLICATIONS RELATIVES A LA FAUNE CAVERNICOLE BULGARE, PARUES DE 1966 à 1970 ET SUPPLÉMENT À LA LISTE DES TRAVAUX PRÉCÉDENTS}

ANDREEV, S. (1970) - Beitrag zur Untersuchung der Landasseln Bulgariens - neue Art der Gattung Trichoniscus (Isopoda, Oniscoidea), Comptes rendus de l'Ac.bulgare des Sciences, 23, 9, pp.1135-1138.

ANDREEV, S. (1972) - Beitrag zur Kenntnis der Landasseln Bulgariens (Isopoda, Oniscoidea). II. Bull.Inst.Zool., Sofia, XXXIV, pp.178-185.

ANDREEV, S. (1972a) - Diffusion du genre Niphargus en Bulgarie et notes taxonomiques sur Niphargus bureschi. Mem. Mus. Civ. St. Nat. Verona, XX, pp.61-66.

ANDREEV, S. (1972b) - Beitrag zur Kenntnis der Landasseln. VII. Trichoniscus valkanovi sp.n. aus Bulgarien. Comptes rendus de l'Ac.bulgare des Sc., Sofia, sous presse.

ANDREEV, S. et TABACARU, J. (1972) - Sur une nouvelle espèce du genre Trichoniscus de Bulgarie, Trichoniscus raitshevi n.sp. (Isopoda, Oniscoidea). Comptes rendus de l'Ac.bulgare des Sciences, Sofia, sous presse. 
ANGELOV, A. (1958) - Sphaeromides polateni ein neuer Vertreter der Höhlenfauna Bulgariens (Isopoda - Cirolanidae). Bull.Inst.Zool., Sofia, XXVII, pp.195-213.

BERON, P. (1968) - Notes sur quelques Acariens des fam. Myobiidae, Spinturnicidae et Macronyssidae, parasites des Chauves-souris en Bulgarie. Bull. Inst. Zool., Sofia, XXVII, pp.157-161.

BERON, P. (1970) - Sur quelques Acariens (Myobiidae, Psorergatidae, Spinturnicidae, Sarcoptidae et Listrophoroidea) de Bulgarie et de l'île de Crète. Bull.Inst.Zool., Sofia, XXXII, pp.143-149.

BERON, P. et GUÉORGUIEV, V. (1967) - Essai sur la faune cavernicole de Bulgarie.II. Résultats des recherches biospéléologiques de 1961-1965. Bull.Inst.Zool.Sofia, XXIV, pp.151-212.

BESHOVSKY, V. (1972) - Representatives of Diptera Brachycera in the caves of Bulgaria. Bull.Inst.Zcol. Sofia, XXXV, pp.22-28.

CASSAGNAU, P. (1968) - Les espèces européennes du genre Bilobella (Collemboles Neanuridae). Bull.Mlus.Hist.nat., Paris, 40, 2, pp.292-307.

COIFFAIT, H. (1967) - Quedius nouveaux ou mal connus. Bull.Soc.Hist.Nat.Toulouse, 103, $3-4$, pp.391.424.

COIFFAIT, H. (1969) - Pselaphides balkaniques. Biologia Gallo-Hellenica, II, 1, Toulouse, pp.65-67.

COIFFAIT, H. (1969a) - Quedius nouveaux. $5^{\mathrm{e}}$ Note sur le genre Quedius, Bull.Soc. Hist. Nat. Toulouse, $105,1-2$, pp.44-54.

COIFFAIT, H. (1970) - Un remarquable Duvalius cavernicole nouveau de Bulgarie. Ann. de Spéléol., 25, 3, Toulouse, pp.721-723.

CVETkOV, L. (1967) - Au sujet de la faune des Stenasellus de la Péninsule balkanique et sur l'origine des Stenasellus. Bull.Inst.Zool., Sofia, XXIII, pp.139-165.

DELČEV, H. (1967) - On the studies of spiders (Araneae) in the Vitosha Mountain. Bull.Inst.Zool., Sofia, XXIV, pp.51-56.

DELČEV, H. (1970) - Neue Daten über die Verbreitung der Gattung Meta (Araneae, Araneidae) in Bulgarischen Höhlen. Bull.Inst.Zool., Sofia XXXII, pp.89-92.

DELTSHEV, H. (197?) - A contribution to the study of spiders (Araneae) from the caves in Bulgaria. Bull.Inst.Zool., Sofia, XXXIV, pp.171-175.

DELTSHEV, H. (197:a) - A contribution to the study of spiders (Araneae) from the caves in Bulgaria. II. Genus Lepthyphantes in Bulgarian caves. Bull.Inst.Zool., Sofia.

DELTSHEV. H. (1972b) - A new genus of Bulgarian cave spiders Protoleptoneta bulgarica n.g., n.sp., leeptonetidae. Int. J. Speleol., 4, pp. 275-283.

DUMITRESCO, M. (1971) - Une Araignée nouvelle des grottes de Bulgarie, Antrohyphantes rodopicus a.g., n.sp. (fam. Linyphiidae, sous-fam. Linyphiinae, série de genres Lepthyphanteae). Trav.Inst.Spéol., Bucarest, 10, pp.167-174.

DUMITRESCO, M. et GEORGESCO, M. (1970) - Révision des représentants du genre Troglohyphantes des grottes de Roumanie. Livre de centenaire d'Emile G.Racovitza, Bucarest, pp.313-331.

DUMITRESCO, M. et ORGHIDAN, T. (1969) - Date noi obtinute in studiul faunei litoclazice. Lucr.Inst.de Speol. "Emil Racovita", VIII, pp.55-71.

GUÉORGUIEV, V. (1969) - La répartition des Coléoptères troglobies en Bulgarie. Actes du IV ${ }^{\mathrm{e}}$ Congres Intern. de Spéléologie en Yougoslavie (12-26 IX 1965), 4-5, Ljubljana, pp.109-112.

GUÉORGUIEV, V. (1971) - Notes sur le sous-genre Paraduvalius Kn. et descriptions de deux espèces nouvelles (Coleoptera, Carabidae - genre Duvalius). Bull.Inst.Zool., Sofia, XXXIII, pp.155-164.

GULIČKA, J. (1967) - Neue und interessante Diplopoden aus Bulgarien. Annotationes Zool. et Bot., Bratislava, 39, pp.1-9.

GULIČKA, J. (1969a) - Neue Höhlen-Diplopoden aus Bulgarien. Annotationes Zool. et Bot., Bratislava, 42, pp.1-5.

HAZELTON, M. (1970) - Fauna from some caves in Bulgaria \& one in Yugoslavia. The Trans.Cave Res. Group of Gr. Britain, 12, 1, Ledbury, pp.33-37. 
HURKA, K. (1969) - Systematic, faunal and bionomical notes on the european and asiatic flea species of the family Ischnopsyllidae (Aphaniptera). Acta Univ. Carolinae, Biologia, pp.11-26.

KARAMAN, Z. (1969) - Über einige neue balkanische Pselaphiden (Col.). Biologia GalloHellenica, Toulouse, II, 1, pp.49-63.

KARAMAN, Z. (1969a) - Über einige neue und interessante Pselaphiden Vertreter der Balkanhalbinsel. Fragmenta Balcanica, Skopje, VII, 3(161), pp.9-20.

KOLEBINOVA, M. (1967) - Faunistic studies on some Acari of the families Myobiidae and Cheyletidae, parasitizing on small Mammals in Bulgaria. Bull.Inst.Zool., Sofia, XXV, pp.73-77.

KOLEBINOVA, M. (1968) - Neotrombicula (Neotrombicula) rhinolophi - a new species parasitic on Bats in Bulgaria (Acarina: Trombiculidae). Comptes rendus de l'Ac. bulgare des Sciences, Sofia, 21, 4, pp.383-386.

KUMANSKI, K. (1968) - Beitrag zur Erforschung der Trichopteren Bulgariens (I). Faun. Abh., Dresden, 2, 16, pp.109-115.

KUMANSKI, K. (1971) - Beitrag zur Untersuchung der Köcherfliegen (Trichoptera) Bulgariens. III. Bull.Inst.Zool., Sofia, XXXIII, pp.99-107 (en bulgare, rés. en russe et en allem.).

KUMANSKI, K. (1972) - Beitrag zur Erforschung der Köcherfliegen (Trichoptera) Bulgariens. IV. Vertreter aus bulgarischen Höhlen. Bull.Inst.Zool., Sofia, XXXIV, pp. 209-216.

KWARTIRNIKOV, M. (1971) - Untersuchungen über Quedius mesomelinus Marsch. (Coleoptera, Staphylinidae). Bull.Inst.Zool., Sofia, XXXIII, pp.29-36.

MATIC, Z. (sous presse) - Nouvelles contributions à la connaissance des Chilopodes cavernicoles de Bulgarie. Bull.Inst.Zool., Sofia, XXXVIII.

MATIC, Z. et DARABANTZU, C. (sous presse) - Contributions à la connaissance du genre Thracophilus Verh. (Geophilomorpha - Himantariidae).

MATIC, Z. et GOLEMANSKY, V. (1967) - Recherches sur les espèces et l'écologie des Lithobiidae (Chilopoda, Lithobiomorpha) en Bulgarie. Bull.Inst.Zool., Sofia, XXIV, pp.121-132.

MIHAILOVA-NEIKOV A, M. (1969) - Elaphoidella angelovi n.sp. from a cave in the Rhodopes Mountains. Ann.Univ. Sofia, Fac.Biol., Livre 1, 62, pp.61-70.

POPOV, A. (1969) - La faune cavernicole dans la région du v.Gabare, distr. Vraca. Annuel de Spéléologie, Ed.STD “Akademik", Année 1968, Sofia, pp.36-40 (en bulgare, rés. en français, p.43).

RADOVANOVIC, M. (1953) - Beitrag zur Kenntnis der Trichopteren Jugoslaviens. Glas SAN, CCX Oddel.prir.nauka, 7, 1, pp.11-36, Beograd.

RAMBOUSEK, Fr.G. (1909) - Sur les Staphylinides de Bulgarie. Vestnik kral. české spol. nauk, II, Praha, pp.1-23.

RIEDEL, A. (1967) - Daudebardiinea (Gastropoda, Zonitidae) Bulgariens, Annales Zoologici, Warszawa, XXIV, 8. pp.463-483.

RIEDEL, A. (1969) - Die Untergattung Morlina A.J.Wagner und Riedelius Hudec der Gattung Oxychilus Fitzinger (Gastropoda, Zonitidae) Annales Zoologici, XXVII, 6, Warszawa, pp.91-131.

SKALSKI, A. (1971) - Note on Lepidoptera from Bulgarian Caves. Intern. J. of Speleol., III, 3-4, pp. 215-217.

SLIVOV, A. (1968) - Schmetterlinge (Lepidoptera) von Iskârdurchbruch. I. Macrolepidoptera. Bull. Inst. Zool., Sofia, XXVIII, pp.195-186

STRASSER, K. (1969) - Über Diplopoden Bulgariens, II. Annales Zoologici, Warszawa, XXVII, 7, pp.133-168.

TABACARU, I. (1967) - Beiträge zur Kenntnis der cavernicolen Antroleucosomiden (Diplopoda, Ascospermophora). Int. J. Speleol., III, pp.1-31.

TURK, F.A. (1970) - Some notes on the Acari and Myriapoda collected by Dr.P.Brown and Mr. W.G.R.Maxwell in Bulgarian caves. The Trans. Cave Res. Group of Gr.Britain, Ledbury, 12, 1, pp.39-41. 
URBANSKI, J. et WIKTOR, A. (1967) - Beiträge zur Kenntnis bulgarischer Nacktschnecken (Moll., Pulm.)(Systematische, zoogeographische und ökologische Studien über die Mollusken der Balkan-Halbinsel.VIII). Bull. de la Soc. des Amis des Sc. et des Lettres de Poznan, Sér.D, 8 Livr. (1967), pp.47-95.

VANDEL, A. (1967) - Les Isopodes terrestres et cavernicoles de la Bulgarie (seconde partie). Ann. Spéléol., XXII, 2, pp.333-365.

Anonyme [Horaček, Iv. et al.] (1971) - [Contribution à l'étude des petits Mammifères des Rhodopes], Rodopsk pešternjak, VII, 54, pp.40-44 (en bulgare).

\section{RESUMÉ}

Le présent travail fait suite à deux travaux précédents sur la faune cavernicole bulgare (1962 et 1967). Les trois englobent des données sur 431 cavités karstiques bulgares, plus ou moins prospectées du point de vue biospéléologique.

On trouvera ici une énumération de 147 grottes et gouffres nouveaux et de 293 espèces animales, dont 154 ne sont pas mentionnées dans les deux listes précédentes. Une bibliographie, comprenant 55 titres, y est ajoutée.

\section{ZUSAMMEN FASSUNG}

Die vorliegende Arbeit ist eine Fortsetzung der beiden früheren Aufstellungen der bulgarischen Höhlenfauna (1962 und 1967). Die drei Aufstellungen enthalten Angaben über 431 Höhlen in Bulgarien, die mehr oder weniger in biospeläologischer Hinsicht erforscht worden sind.

In dieser Aufstellung sind 147 neue Höhlen und Schächte angegeben, sowie 293 Tierarten, wovon 154 Arten in den beiden früheren Aufstellungen nicht erwähnt wurden. Dazu kommt ein Literaturverzeichnis mit 55 Titeln. 